\title{
Literature Review of Early Childhood Music Therapy Between 1990-2012
}

\author{
Kirsi Tuomi ${ }^{12}{ }^{*}$, Esa Ala-Ruona ${ }^{1}$, Amelia Oldfield ${ }^{2}$ \\ 1 University of Jyväskylä, Finland \\ 2 Anglia Ruskin University, United Kingdom \\ *kirsit@yahoo.com \\ Received: 21 July 2016 ; Accepted: 20 May 2017 ; Published: 1 July 2017 \\ Editor: Seung-A Kim Reviewers: Elizabeth Schwartz, Jane Edwards
}

\begin{abstract}
The article examines music therapy literature from 1990 to 2012 focusing on children aged 0 to 5 -years old. The literature includes clinical descriptions, research articles, chapters in books, peer reviewed electronic publications, and peer reviewed journals. Altogether 125 different texts were found which fulfilled the criteria for inclusion. Simple quantitative analysis gave guidelines for deeper, comparative qualitative analysis. According to the data the older children were more often written about than younger children. Historically the dominance from individual work has been shifting to dyadic/family work. The active methods were most commonly singing and playing with instruments. Children with autism spectrum disorder (ASD) were most strongly represented together with paediatric patients and children with developmental disabilities. Interaction between family members and the positive factors were emphasized in the articles. The importance of fun and enjoyment was underlined throughout all client groups. The results claim that more specific information of the effects of early childhood music therapy is needed. In addition, related areas of research, cross-scientific viewpoints, and common fields of interests should be taken into consideration in the future.
\end{abstract}

Keywords: music therapy, young children, early childhood, literature review

\section{Introduction}

Early childhood is undoubtedly the phase of life when the basics of many developmental cornerstones are laid. The impact of the early years for the later life is undisputed (Leckman \& March, 2011; Roth \& Sweatt, 2011; Schore, 1994; 2014). When the development of the child is somehow atypical or at risk, it is logical that early interventions are more effective, even in terms of funding than later interventions - to put it simply: the earlier the intervention is made, the less entrenched the difficulties that will need treatment will be (Golos et al., 2011; Hayes et al., 2014; Peters-Scheffera et al., 2012; Reynolds et al., 2011; Santelices et al., 2011).

Infant, toddler, pre-schooler, and early childhood may refer to children of different ages. This might be because of cultural differences but also the lack of strict definitions. Definitions of the age of an infant varies from 0-12 months (Medicinenet.com) to just "a very young child or baby" (English Oxford Living Dictionaries). Toddler is described as a young child who is learning to walk (Medicinenet.com; English Oxford Living 
Dictionaries). Infant and toddler may in some cases be used as synonyms (Gilliam \& Mayes, 2005; Shonkoff et al., 2005), as well as toddler and preschooler (Egger, 2009). In addition, children start school at different ages and childcare arrangements may vary greatly from one country to another, which may impact the terminology used.

"Early childhood music therapy" is a relatively new phrase. Schwartz referred to children aged 0-5 when writing about music, therapy, and early childhood (Schwarz, 2008) and the same age group was brought up by Kern and Humpal (2012) in their book Early Childhood Music Therapy and Autism Spectrum Disorders. This terminology has its place in the same way as: "early childhood education" and "early childhood music education," for example. Also, the first international publication from this field is called Imagine - online Magazine of Early Childhood Music Therapy (http://imagine.musictherapy.biz/Imagine/home.html). In the present article, the authors will use the terminology early childhood music therapy to refer music therapy with children from birth to age 5.

When the target is to improve clinical efficacy and inform future research guidelines, the concept of understanding the phenomena is important (Dileo, 2005; Randolph, 2009). Understanding the nature of early childhood music therapy is possible by exploring the literature that represents developments in the field worldwide. The review undertaken for this paper focuses on how an early childhood music therapy intervention has been applied and how this practice is carried out (Randolph, 2009). In addition, its aim is to refine and conceptualize the field and simultaneously identify gaps in the literature (Dileo, 2005). According to the writers' knowledge, no such research has been accomplished before. The article is a part of the first author's doctoral study conducted at the University of Jyväskylä, Finland in collaboration with Anglia Ruskin University, UK. The intention has been to analyse the literature as objectively as possible with the understanding that the authors' music therapy background, experience, education, and philosophical outlooks will influence the interpretations made.

\section{Data and prerequisites for the data}

This review was intended to be a descriptive, not a systematic review. However, it was a detailed and careful study of existing resources, which the authors believe will be of value to other music therapists. As Aveyard (2010) pointed out such a review can be conducted in a systematic manner even if the detail required for systematic reviews was not attained.

The data included was broad, versatile, and diverse. The researchers were interested in the early childhood music therapy field as a whole, including theory, practice, and research studies. Case studies written by music therapists were included as well as general descriptions of music therapists' clinical approaches. At a further stage of analysis, the texts were divided up into different categories so it is possible to see which articles were research based. The different categories used will be clearly defined when that data is presented.

Included texts present clinical early childhood music therapy work and early childhood music therapy research in any or all the following: books, peer reviewed electronic publications, and peer reviewed journals from 1990 to 2012. Literature was limited to accessible publications in the English language. The following publications were included in the systematic search: Music Therapy, Journal of Music Therapy, Music Therapy Perspectives, Nordic Journal of Music Therapy, British Journal of Music Therapy, and Australian Journal of Music Therapy (online availability from year 2005 from which the articles included). In addition, CDROMS Music Therapy Today and Music Therapy World were searched through. Articles published elsewhere were searched with different keywords defining early childhood music therapy such as music therapy + early childhood / small children / young children / early intervention. This search was concluded with multidisciplinary searches in electronic databases including different medical and psychiatric databases (for example PsychInfo and PubMed). The books and book chap- 
ters were searched manually in the libraries of the same universities. Reference lists in the papers were searched to find additional texts.

In several cases the line between music therapy, music education, cognitive music research, psychology of music, and different supportive music approaches was challenging to draw. The aim of the research was to focus specifically on music therapy. For the purposes of this review music therapy is defined as "a systematic process of intervention wherein the therapist helps the client to promote health, using music experiences and the relationships that develop through them as dynamic forces of change" (Bruscia, 1998, p. 20). In addition to Bruscia's definition the interactional dimension of music therapy was stressed. Music therapy is understood as a process where attunement to the client is possible by adapting to the present moment and the needs of the client (Oldfield, 2006, 2017; Stern, 2010; Tuomi, 2017). Because of the strict definition, some excellent approaches and research papers (Cassidy \& Ditty 2001; Kern 2006; Lim 2010) were excluded.

Criteria for inclusion were:

- The article was published for the first time between 1990-2012;

- The definition of music therapy as understood above was fulfilled;

- Music therapy as such was mentioned in the article either in titles, abstract, or text;

- At least one of the cases concerned children under 6 years of age; and

- Clinical music therapy could be detected in some way within this age group

Criteria for exclusion were:

- Theses and dissertations;

- Conference papers;

- Review articles and duplicates; and

- Theoretical articles, which did not include any clinical examples.

In the case of edited books, each chapter was treated independently. If one author had written the entire book it was identified as one whole.

Premature infants and music therapy in the Neonatal Intensive Care Unit (NICU) are not included in this article. When collecting data, it was soon revealed that this was a clinical field in its own right. In addition, an integrative review of music therapy conducted with premature infants was already published (Haslbeck, 2012) and also a meta-analysis of NICU music therapy (Standley, 2012).

\section{Analysis methods}

Early childhood music therapy literature from 1990 to 2012 was sourced and examined. The authors focused on how these texts described music therapy interventions and how music therapy services and interactions in sessions occurred. The analysis is further described below.

In total, 125 articles, books, and book chapters were found. The titles and details of each publication was entered Excel to create an annotated bibliography of the whole data. Initially obvious categories such as the name of the author, year of the publication, name of the publication and the genre of the text, for example research or clinical description, were included. When the analysis proceeded the number of categories increased and categories such as the age of children, the names of clinical population, for example child with autism spectrum disorder (ASD), patients in hospice, at-risk families, and the type of therapy context, for example individual, group, family, were added. Later it became clear that more categories such as the amount of cases and a historical viewpoint would improve the detail provided. The preliminary categories were therefore specified during the process as were the excluding and including attributes. For each article the following data was included in the Excel table in a separate column (Table 1).

Simple analysis of the bibliography including detailing how many texts were about individual music therapy, how many were accomplished with 4-year-old children, how 
Table 1. An example of data in Excel table

\begin{tabular}{|c|c|c|}
\hline Authors & Year & Name of the article \\
\hline Aasgaard, T. & 2002 & $\begin{array}{l}\text { Musical Acts Of Love In The Care Of Se- } \\
\text { verely Ill and Dying Children and Their } \\
\text { Families }\end{array}$ \\
\hline Aasgaard, T. & 2005 & $\begin{array}{l}\text { Song Creations by Children with Cancer - } \\
\text { Process and Meaning }\end{array}$ \\
\hline Abad, V. & 2007 & $\begin{array}{l}\text { Early Intervention Music Therapy: Report- } \\
\text { ing on a 3-Year Project to Address Needs } \\
\text { with At-Risk Families. }\end{array}$ \\
\hline Abad, V. \& Williams, K. & 2006 & $\begin{array}{l}\text { Early Intervention Music Therapy for } \\
\text { Adolecsent Mothers and their Children }\end{array}$ \\
\hline Achenbach, $\mathrm{C}$. & 2012 & $\begin{array}{l}\text { Nordoff-Robbins Music Therapy in a Nurs- } \\
\text { ery Setting. Supporting Music Therapy } \\
\text { Students On Placement }\end{array}$ \\
\hline Aldridge, $\mathrm{K}$. & 1993 & $\begin{array}{l}\text { The Use Of Music To Relieve Pre-Opera- } \\
\text { tional Anxiety In Children Attending Day } \\
\text { Surgery }\end{array}$ \\
\hline Bargiel, M. & 2004 & $\begin{array}{l}\text { Lullabies And Play Songs. Theoretical } \\
\text { Considerations For An Early Attachment } \\
\text { Music Therapy Intervention Through } \\
\text { Parental Singing For Developmentally A- } \\
\text { Risk Infants }\end{array}$ \\
\hline
\end{tabular}

many of them addressed music therapy with ASD children, and these details prompted guidelines for further qualitative analysis.

Descriptive analysis of the effects of music therapy was accomplished with the help of mind-map techniques in CMapTools (CMap, 2017). Preliminary categories were formed on the bases of the writers' clinical expertise. All texts were read through by analysing the content and categorized first under preliminary categories. The categories developed and expanded while new findings emerged. Several subcategories were developed and careful notes were made. Different categories were sorted again by combining and developing division of the categories. The tables were made to help gain deeper insight (Galvan, 2009).

In many cases the articles belonged to several different categories at once. When these overlaps were revealed the articles were listed as many times as required, twice or even more. This applied to both quantitative and qualitative analysis.

\section{Results}

When interpreting the tables in the results section the authors make an assumption that the amount of texts published corresponds to some degree with the amount of clinical work occurring in the text. The authors are aware that there might be instances where this assumption is incorrect.

\section{Age of children and type of therapy}

When looking at the data from the perspective of age distribution it seems that the line is rising (Figure 1). Apart from infants under 1 year, the data reveals that there are more articles the older the children are. 


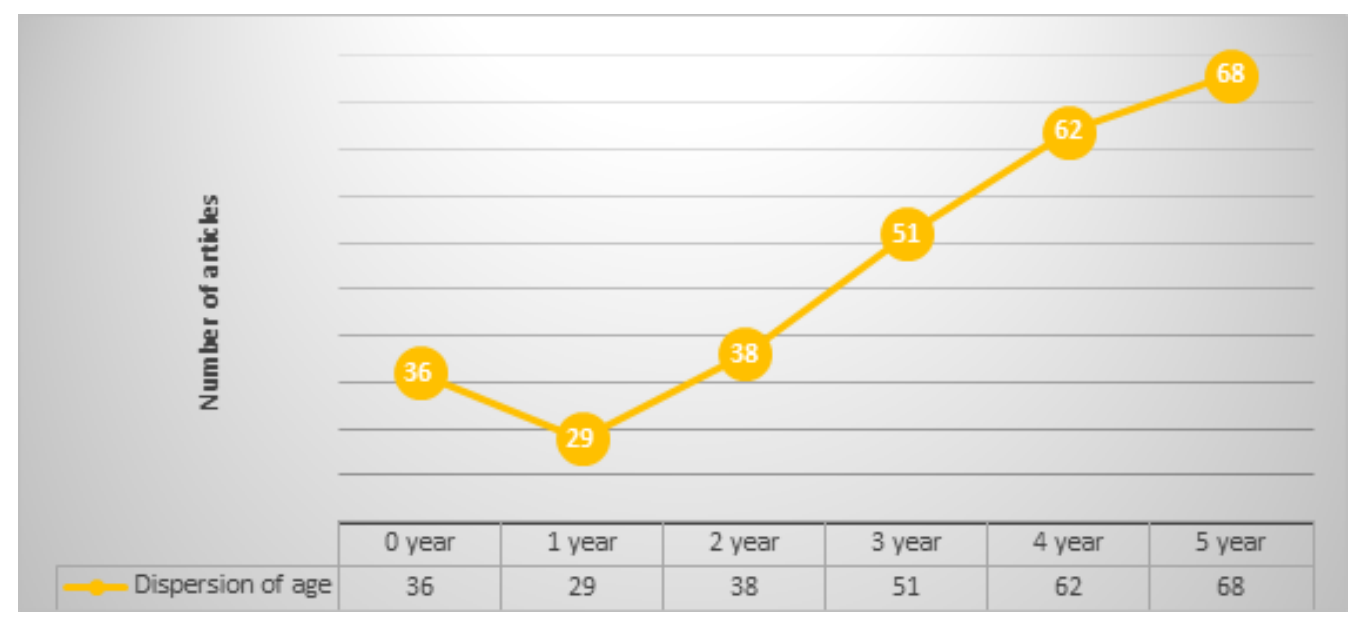

Figure 1. Distribution of age

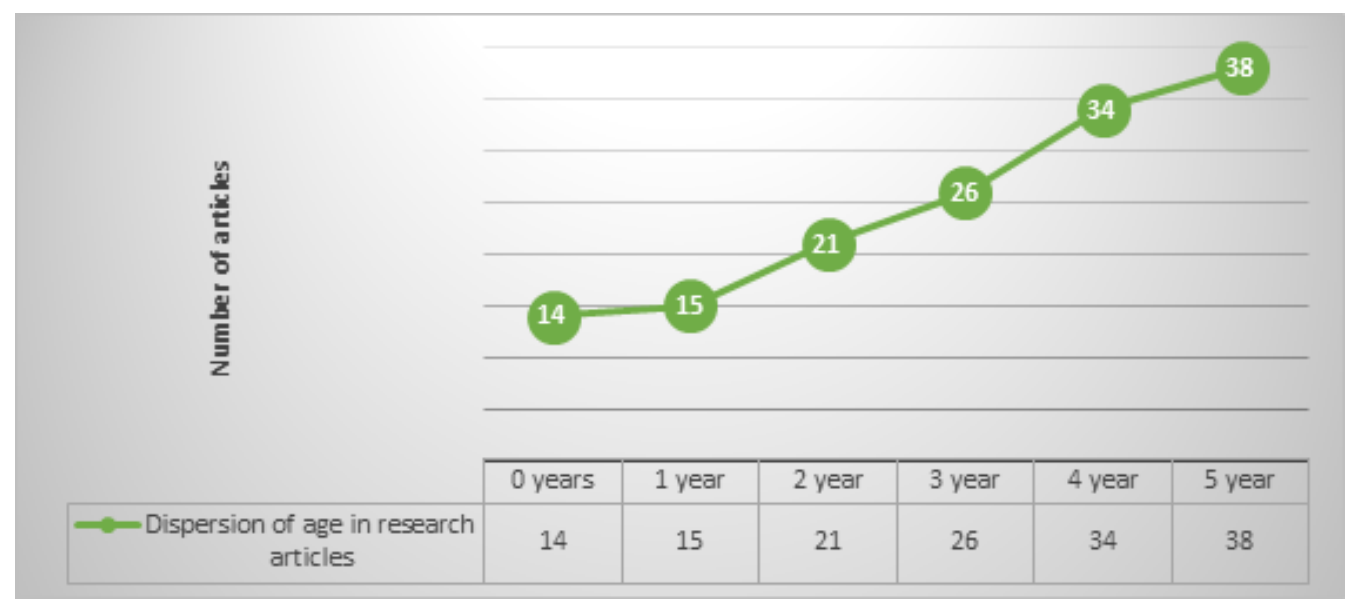

Figure 2. Distribution of age in research articles

One-year-old children were a minority age group in the music therapy literature. The difference between written texts between 1 and 5-year-old children was 39 texts, which is quite a lot when examining all the data.

The same tendency seems to be present when viewing the research data (Figure 2). There is clearly less research with toddlers from 0 to 2 years old than with children of 5 years of age.

The type of therapy was divided into three: individual, group, and dyadic/family work. Dyadic work was defined as work where the music therapist worked with the child and a carer. Often the carer would have been the parent but it also could have been a relative, a foster carer, a nursery nurse, or a member of care staff. As can be seen in Figure 3 it seems that the amount of individual and dyadic/family work is almost equal.

Each category is not completely separate from the other, as group work sometimes overlaps with dyadic/family work, because the groups could be for individual children and also for dyads or families. In addition, in numerous cases the same text introduces several different types of therapy. As a result, some texts are included several times in the data analysis.

When looking at research data the results seem to be somewhat different (Figure 4). The differences between types of work are smaller and group work seems to be researched most often.

Sing\&Grow (Williams \& Abad, 2005) was founded in Australia. It is a group based 10 -week program aimed at families with young children. The program was funded for 2 years initially and then the funding was extended. In the findings of this review 


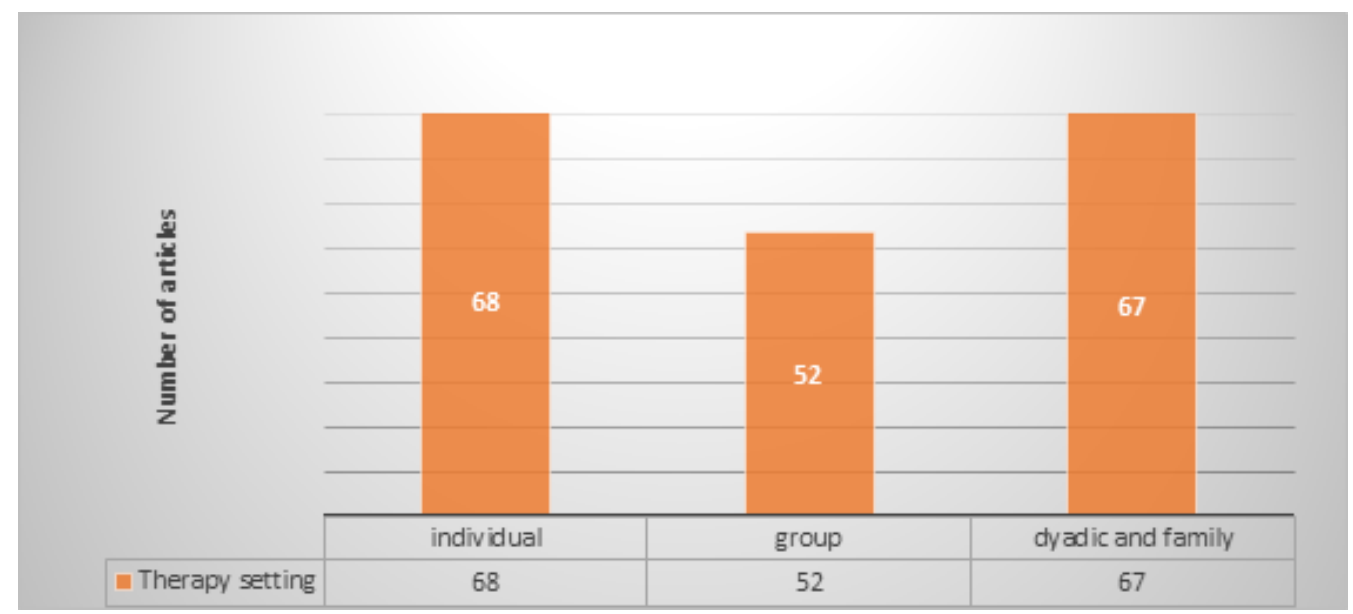

Figure 3. Therapy types

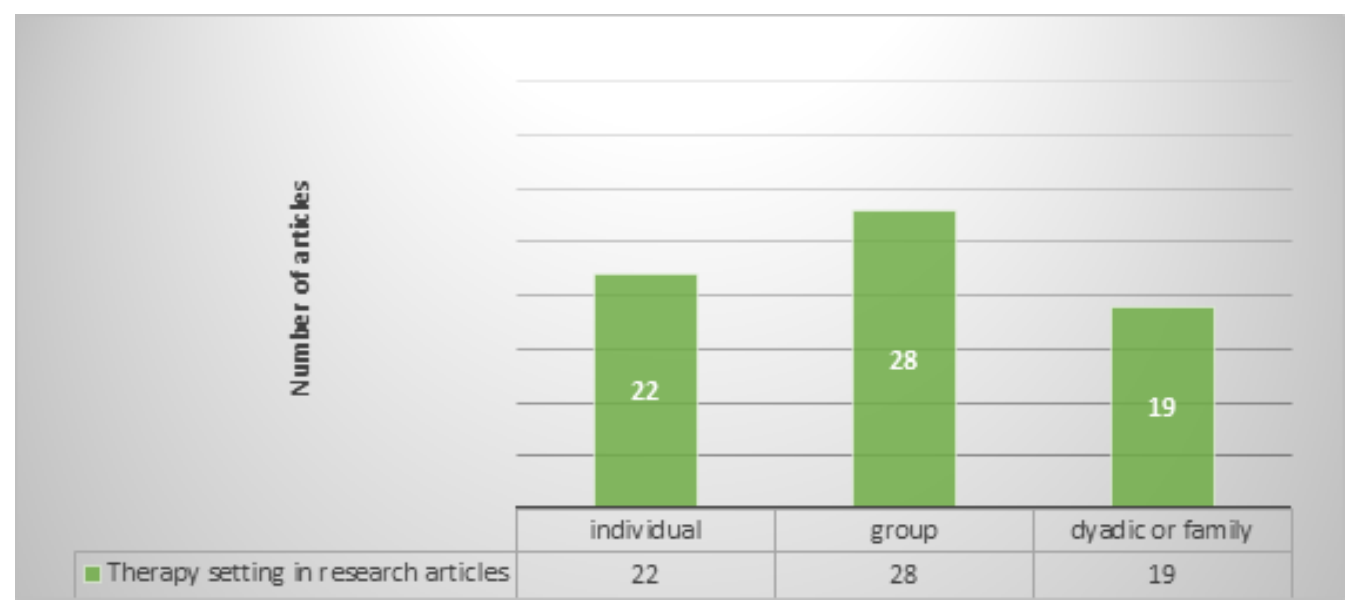

Figure 4. Therapy types in research articles

five Sing\&Grow research papers were included (Abad, 2007; Abad \& Williams, 2006; Nicholson et al., 2010; Nicholson et al., 2008; Williams et al., 2012) and one clinical evaluative paper (Williams \& Abad, 2005). These have affected especially the analysis results of research articles. If the Sing\&Grow papers would had been excluded from the data the amount of research texts would have been quite close to each other when comparing individual and group work. On the other hand, the family work would had been rated lower.

It was interesting to find out if the age of child had had an influence on whether work was carried out individually, in a group, or with the family. The analysis showed (Figure 5) that during the first year including the carer in therapy was almost as common as it was with 5-year-old children. Though, there were 17 articles describing individual music therapy for babies. When different types of therapy were quite the same in children with 3 years of age, the individual music therapy was clearly the most common therapy type with 5-year-old children. Again, it must be pointed out that group work was conducted with families as well as with individual children.

\section{Historical viewpoints}

The number of early childhood music therapy articles increased annually between 1990 and 2012. The time brackets analysed were either 5 or 6 years long except for the last one, which was only 2 years long because that was when the data gathering stopped. This somewhat affects conclusions made. Between 1990 and 1995, 18 articles were found whereas between 2006 and 2010, 43 articles were found. Between 2011 and 2012, 40 articles were found. 


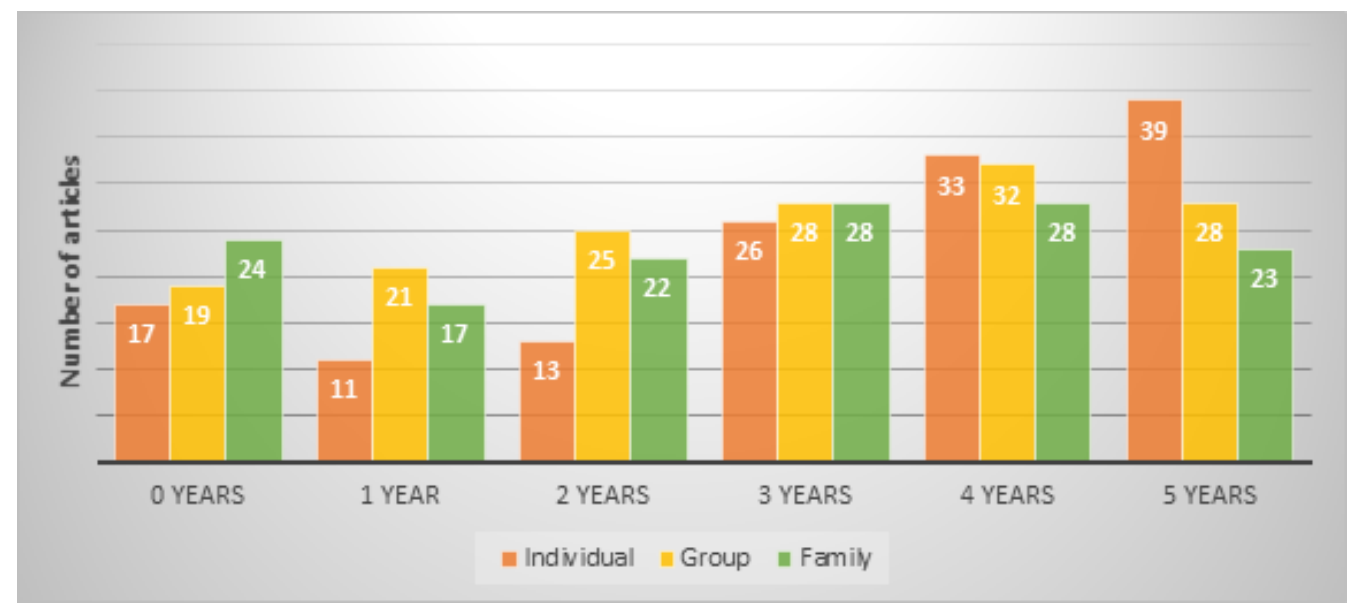

Figure 5. Types of therapy compared with age of children

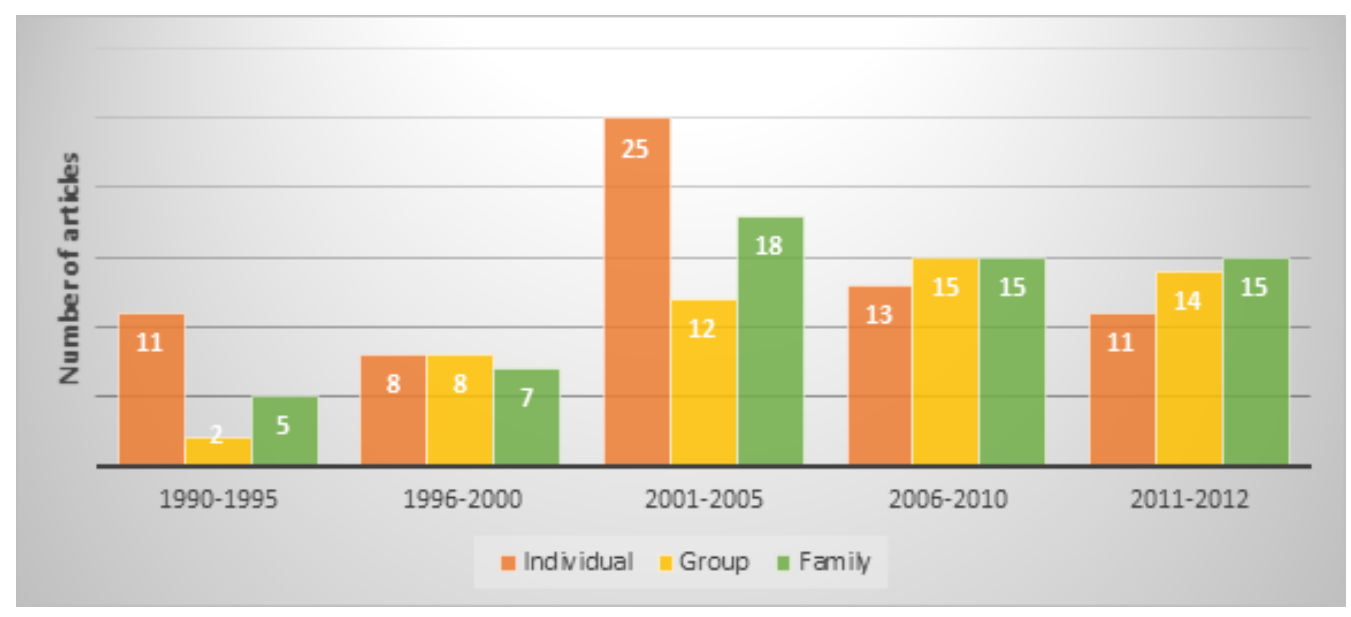

Figure 6. Historical perspectives and therapy types

When having a closer look at historical viewpoints and types of music therapy (individual, group, and family) mentioned during these 22 years it seems there have been changes not only in the amount of texts but also in the types of therapy presented. In Figure 6 it can be seen that individual work was dominant from 1990 to 1995 and between 2001 and 2005. Since then, group work and family work have been more prevalent, and during 2011 and 2012 it seems that dyadic/family approaches have been the area most written about.

\section{Music Therapy techniques used in therapy}

The data was divided into active, receptive, and active-receptive categories. Nine texts could not be included because the relevant information was not in the text. For the rest of the material (116 texts) the analysis revealed that active methods were most common. Seventy-five percent reported using singing, playing, improvising, or other kinds of active methods. Twenty-two percent of texts described using both active and receptive methods and only three percent used solely listening and other receptive methods. In Figure 7 it can be seen that singing was used in over 90 percent of the data detected. Playing with instruments was also very commonly used. Only a little over half of the texts mentioned using improvisation.

\section{Client groups}

There were some difficulties when categorizing the client groups in the data. It seemed that the categories could be grouped in many ways and the categorizing system was 


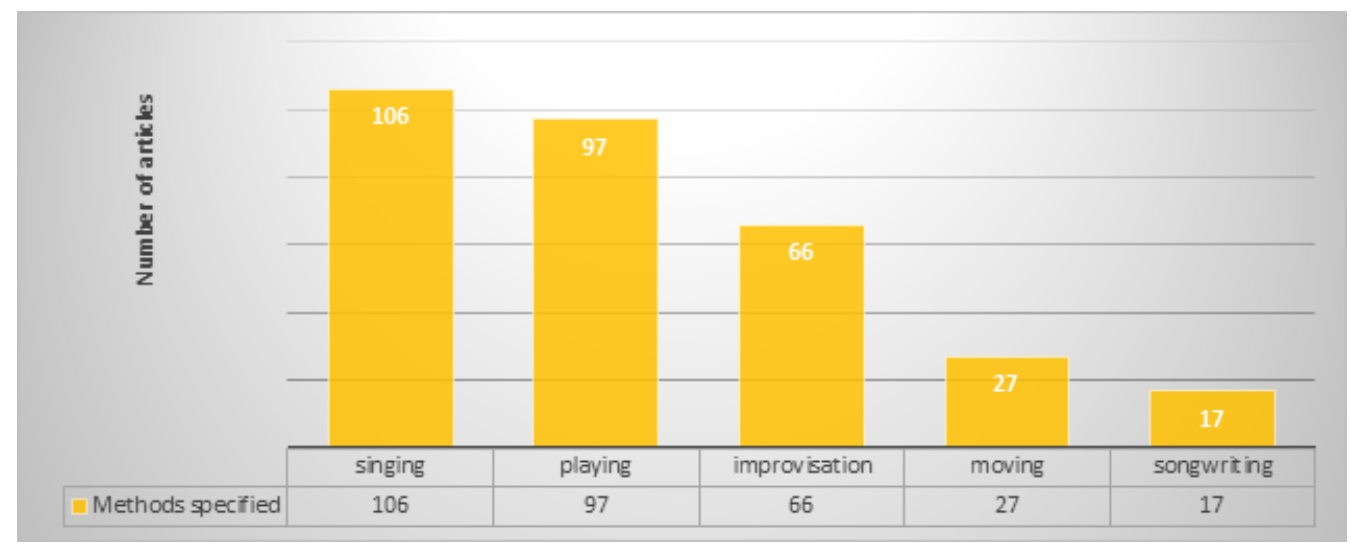

Figure 7. Therapy methods specified

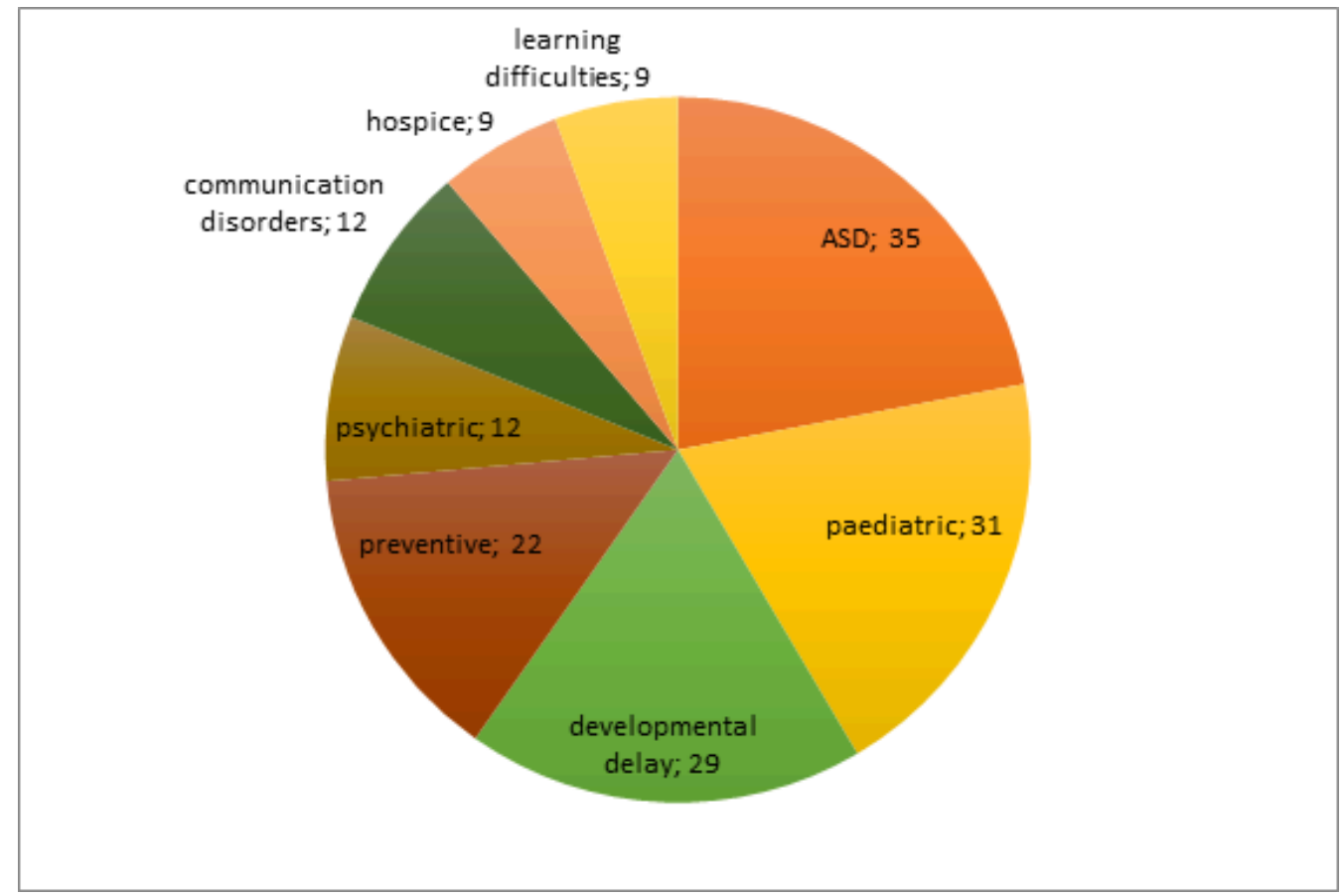

Figure 8. Client groups

different in different parts of the world. For example, ASD could be seen under communication disorders as well as under developmental delays while developmental delays and learning disabilities could be seen as the same category.

The categories were finally developed after sorting out and analysing the data by following the definitions of the writers. The following categories were decided upon: ASD, developmental delays, paediatric patients, preventive approaches, psychiatric disorders, communication disorders, hospice patients, learning difficulties, attachment issues, sensory impairment, and parenting issues. However, it should be pointed out that because of geographical divergence the terms might be presented differently in different texts. Due to this fact, there may be overlaps among categories and therefore categorizing should be seen as a rough idea rather than a definite grouping.

When looking at the client groups in Figure 8, it reveals that children with ASD are most strongly represented. When adding clients from paediatrics it seems that these client groups cover a little over half of the whole data. Children with developmental disabilities and delays and preventive music therapy approaches were quite well represented, whereas the psychiatric field and children with communication disorders were in the minority. Few texts referred to hospice clients, learning difficulties, sensory impairments, parenting, and attachment issues which can be found later in Table 1. 


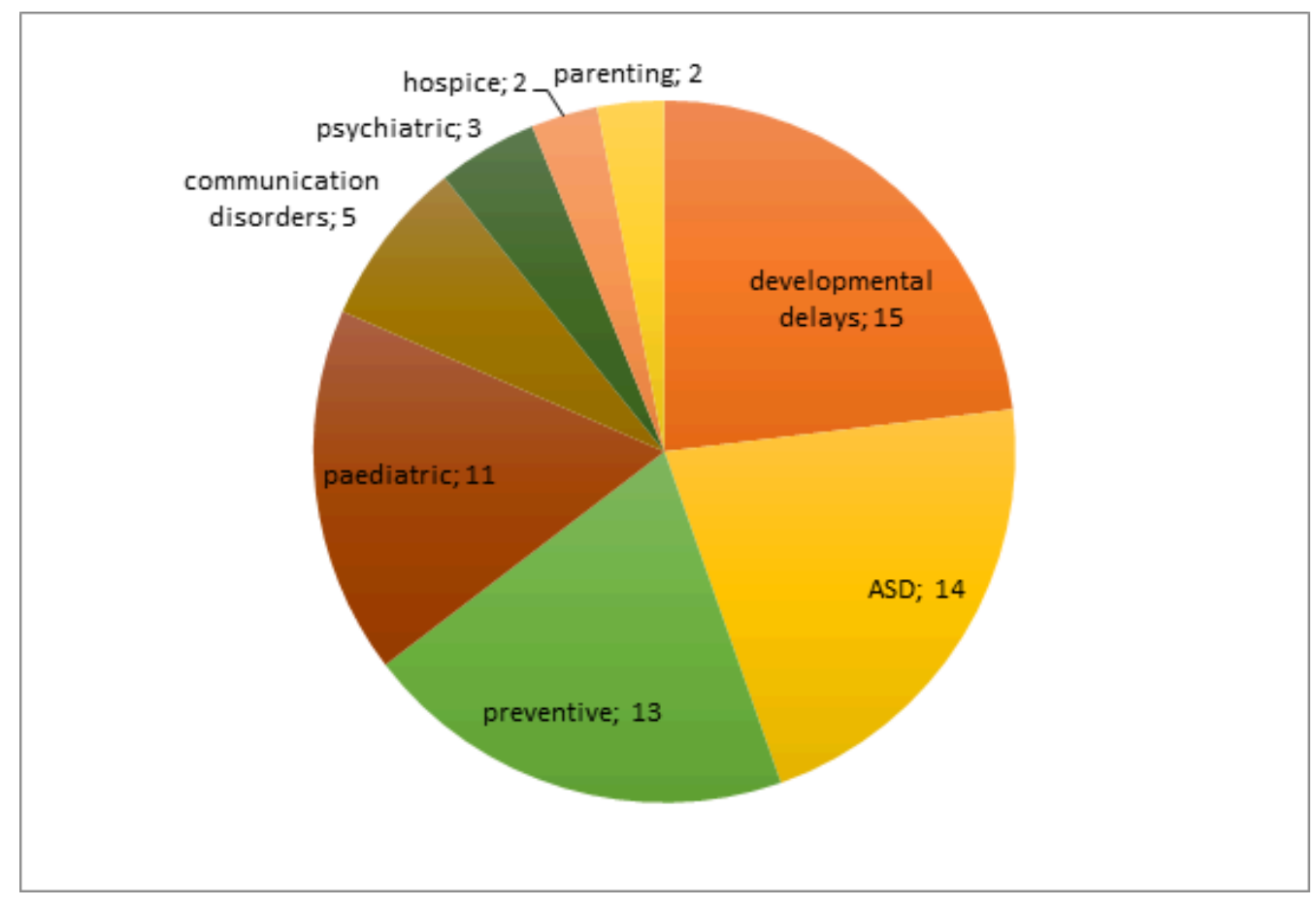

Figure 9. Client groups in research articles

When looking at the different client groups in the research data (Figure 9), the same tendency can be detected. Developmental disorders and delays together with ASD are the most common client groups followed by preventive treatment and paediatric clients.

In the following table (Table 2) the texts are categorized by their client groups. Again, the selected categorizing might affect the results but gives guidelines for readers to find adequate references for their purposes.

\section{Effects of music therapy}

When analysing the data from the viewpoint of reported effects of music therapy, authors mentioned effects in four different areas: a) social areas, b) emotional areas, c) physical and academic skill areas, and d) other areas. Effects in social and emotional areas were written about most, whereas physical and academic skill areas were mentioned less often.

When analysing social areas, the following subcategories emerged:

- Interaction within family,

- Other interaction,

- Communication, and

- Social behavior

The amount of the prominent themes defined the order of listed subcategories. This means that interaction within families was pointed out most often. In the same table, it can be seen that interactions between family members were emphasized and parenting skills were also quite well represented. Conversely, supporting attachment was mentioned in only four texts and research in this field was completely lacking. Similarly, affect attunement was focused on in only one text (Levinge, 2011) even though it is known that attunement and synchronicity are crucial for well-functioning interaction.

Increased intentions and initiatives, engagement as well as turn-taking and reciprocity emerged quite strongly from the data. Non-verbal interaction was only mentioned in a few texts (Kim et al., 2008; Levinge, 2011; Oldfield, 2011; Skewes \& Thompson, 1998). 
Table 2. References categorized by client groups

\begin{tabular}{|c|c|c|}
\hline Client group & Research references & Other references \\
\hline ASD (35) & $\begin{array}{l}\text { Allgood, 2005; Dellatan, 2003; Finnigan \& } \\
\text { Starr, 2010; Guerrero \& Turry, 2012; Holck, } \\
\text { 2004; Kim et al., 2008; Kim et al., 2009; } \\
\text { Lim \& Draper, 2011; Muller \& Warwick, } \\
\text { 1997; Oldfield, 2006; Register \& Humpal, } \\
\text { 2007; Standley \& Hughes, 1996; Tomlin- } \\
\text { son, 2010; Walworth et al., } 2009 \text { (14) }\end{array}$ & $\begin{array}{l}\text { Achenbach, 2012; Beer, 1990; Berger, 2002; } \\
\text { Brown, 2002; Bunt, 2002; Carpente, 2012; } \\
\text { Darnley-Smith \& Patey, 2003; Davies \& } \\
\text { Rosscornes, 2012; Humpal, 2012; Jones \& } \\
\text { Oldfield, 1999; Lecourt, 1991; Levinge, } \\
\text { 1990; Lim, 2012; ONeill, 2012; Oldfield, } \\
\text { 2008; Oldfield, 2011; Thompson, 2012; Tre- } \\
\text { varthen et al., 1998; Warnock, 2011; } \\
\text { Wigram, 1995;Woodward, } 2004 \text { (20) }\end{array}$ \\
\hline Paediatric (31) & $\begin{array}{l}\text { Aasgaard, 2005; Aldridge, 1993; Ayson, } \\
\text { 2008; Barrera et al., 2002; Edwards \& Ken- } \\
\text { nelly, 2004; Loewy et al., 2005; } \\
\text { O'Callaghan et al., 2011; Robb, 2000; } \\
\text { Shoemark \& Grocke, 2010; Walworth, } \\
\text { 2005; Walworth, } 2009 \text { (11) }\end{array}$ & $\begin{array}{l}\text { Aasgaard, 2001; Bartram, 1991; Bruce \& } \\
\text { High, 2012; Dun, 1995; Dun, 1999; Dun, } \\
\text { 2007; Edwards, 1994; Hadley, 1996; Kennel- } \\
\text { ly et al., 2001; Loewy, 2004; Lorenzato, } \\
\text { 2005; Nall \& Everitt, 2005; O`Callaghan \& } \\
\text { Jordan, 2011; O`Neill, 2012; Shoemark, } \\
\text { 1999; Shoemark, 2004; Shoemark, 2006; } \\
\text { Shoemark, 2011; Shoemark \& Dearn, 2008; } \\
\text { Voigt, } 2003 \text { (20) }\end{array}$ \\
\hline $\begin{array}{l}\text { Developmental disabilities and delays } \\
\text { (29) }\end{array}$ & $\begin{array}{l}\text { Allgood, 2005; de Mers et al., 2009; Duffy } \\
\text { \& Fuller, 2000; Elefant \& Wigram, 2005; } \\
\text { Gilboa \& Roginsky, 2010; Guerrero \& Turry, } \\
\text { 2012; Holck, 2004; Irgens-Moller, 1999; } \\
\text { Oldfield, 2006; Perry, 2003; Register \& } \\
\text { Humpal, 2007; Standley \& Hughes, 1996; } \\
\text { Sussman, 2009; Williams et al., 2012; } \\
\text { Wylie, } 1996 \text { (15) }\end{array}$ & $\begin{array}{l}\text { Achenbach, 2012; Bruce \& High, 2012; } \\
\text { Darnley-Smith \& Patey, 2003; Hall, 2012; } \\
\text { Jonsdottir, 2002; Loth, 2008; Oldfield, } \\
\text { 2008; Oldfield, 2011; Schwartz, 2008; } \\
\text { Schwartz, 2011; Shoemark, 1996; Skewes \& } \\
\text { Thompson, 1998; Voigt, 2003; Wigram, } \\
1995 \text { (14) }\end{array}$ \\
\hline Preventive (22) & $\begin{array}{l}\text { Abad, 2007; Abad \& Williams, 2006; } \\
\text { MacKenzie \& Hamlett, 2005; Nicholson et } \\
\text { al., 2008; Nicholson et al., 2010; Pasiali, } \\
\text { 2012b; Register, 2001; Register, 2004; Reg- } \\
\text { ister \& Humpal, 2007; Standley et al., } \\
\text { 2009; Standley \& Hughes, 1997; Trolldalen, } \\
\text { 1997; Walworth, } 2009 \text { (13) }\end{array}$ & $\begin{array}{l}\text { Achenbach, 2012; Bargiel, 2004; Cunning- } \\
\text { ham, 2011; Davies \& Rosscornes, 2012; } \\
\text { Drake, 2008; Edwards et al., 2007; Kelly, } \\
\text { 2011; Ledger, 2011; Williams \& Abad, } 2005 \\
\text { (9) }\end{array}$ \\
\hline Communication disorders (12) & $\begin{array}{l}\text { Gross et al., 2010; Holck, 2004; Register \& } \\
\text { Humpal, 2007; Robb, 2003; Standley \& } \\
\text { Hughes, } 1996 \text { (5) }\end{array}$ & $\begin{array}{l}\text { Beathard, 2008; Hall, 2012; Horvat \& } \\
\text { O' Neill, 2008; O`Neill, 2012; Oldfield, 1991; } \\
\text { Oldfield, 2011; Schwartz, } 2011 \text { (7) }\end{array}$ \\
\hline Psychiatric (12) & $\begin{array}{l}\text { Gold et al., 2001; Irgens-Moller, 1999; Lay- } \\
\text { man et al., } 2002 \text { (3) }\end{array}$ & $\begin{array}{l}\text { Brackley, 2012; Burke, 1991; Cassity \& Cas- } \\
\text { sity, 2006; Drake, 2011; Hibben, 1992; Hong } \\
\text { et al., 1998; Levinge, 2011; Oldfield, 1993; } \\
\text { Wildman, } 1995 \text { (9) }\end{array}$ \\
\hline Hospice (9) & $\begin{array}{l}\text { Lindenfelser et al., 2008; Lindenfelser et } \\
\text { al., } 2012 \text { (2) }\end{array}$ & $\begin{array}{l}\text { Aasgaard, 2001; Davis, 2005; Nall \& Everitt, } \\
\text { 2005; O'Callaghan \& Jordan, 2011; Rees, } \\
\text { 2005; Sweeney, 2003; Sweeney-Brown, } \\
2005 \text { (7) }\end{array}$ \\
\hline Learning difficulties (9) & Standley \& Hughes, 1996 (1) & $\begin{array}{l}\text { Bruce \& High, 2012; Hall, 2012; Horvat \& } \\
\text { O' Neill, 2008; Jones \& Oldfield, 1999; Loth, }\end{array}$ \\
\hline
\end{tabular}




\begin{tabular}{|l|l|l|}
\hline \multicolumn{1}{|c|}{ Client group } & \multicolumn{1}{|c|}{ Research references } & \multicolumn{1}{c|}{ Other references } \\
\hline & & $\begin{array}{l}\text { 2008; Ó Neill, 2012; Oldfield, 2011; } \\
\text { Warnock, 2011 (8) }\end{array}$ \\
\hline Attachment issues (5) & $(0)$ & $\begin{array}{l}\text { Cunningham, 2011; Drake, 2011; Kelly, 2011; } \\
\text { Levinge, 2011; Ó Callaghan \& Jordan, 2011 } \\
\text { (5) }\end{array}$ \\
\hline Sense impairment (5) & Robb, 2003; Standley \& Hughes, 1996 (2) & $\begin{array}{l}\text { Gfeller, 1990; Horvat \& Ó Neill, 2008; Salas } \\
\text { \& Gonzales, 1991 (3) }\end{array}$ \\
\hline Parenting (4) & Oldfield et al., 2003; Trolldalen, 1997 (2) & $\begin{array}{l}\text { Achenbach, 2012; Williams \& Abad, 2005 } \\
\text { (2) }\end{array}$ \\
\hline
\end{tabular}

General social behavior, including decreased problem behavior, decreased stereotypic behaviour, and acceptance of differences, was also in the minority when examining the effects of music therapy.

In conclusion, it seems that interaction in this data was described in a general way rather than in a more detailed way. It is mentioned that music therapy helps, supports, and creates interactions but more exact information was often not clearly forthcoming.

The analysis of data relating to emotions revealed four subcategories:

- Positive factors

- Supportive factors

- Expressing and regulating qualities

- Other factors

According to this data it seems that early childhood music therapy literature emphasizes the positive factors of music therapy. The importance of fun and enjoyment was underlined throughout all client groups.

Except for preventative interventions the target of music therapy is often to reduce symptoms whatever those symptoms with different client groups are. In this data, this area was clearly taken into account in only one research publication (Gold et al., 2001). Also, very specific areas like emotional synchronicity were rarely mentioned (Kim et al., 2009) and the texts remained commonly on a more general level.

Expressive and regulating qualities of music therapy were quite well represented. Both areas are important for the development of the child and regulation problems often emerge within the population of young children with difficulties. Regulation of emotions was mentioned primarily with pediatric patients (Aldridge, 1993; Ayson, 2008; Barrera, Rykov \& Doyle, 2002; Hadley, 1996; Loewy, 2004) while with children with ASD it was mentioned in two cases (Berger, 2002; Lecourt, 1991), within the psychiatric field twice (Brackley, 2012; Hong et al., 1998) as well and in hospice settings (Lindenfelser et al., 2008; Lindenfelser et al., 2012).

Physical and academic skill areas were visible in only a relatively small amount of papers. For this reason, both of these areas, with 14 and 11 texts, were put together under the same category heading.

Motor abilities and language development were the largest subcategories in these areas. Motor skills were emphasized especially with children with developmental delays (Bruce \& High, 2012; Duffy \& Fuller, 2000; Wylie, 1996) while effects of music therapy in language development were emphasized with children with ASD and children with communication disorders (Gross, Linden \& Ostermann, 2010; Guerrero \& Turry, 2012; Lim, 2012; Lim \& Draper, 2011).

Many of these subcategories were mentioned only once. There was only one paper written from a neurological viewpoint (Shoemark, 2004). Problems with eating were also mentioned only once (Dellatan, 2003). Language development and learning competences were more frequently written about but pre-writing and -reading compe- 
Table 3. Social areas

\begin{tabular}{|c|c|c|}
\hline Social areas & Definition & Reference \\
\hline $\begin{array}{l}\text { Interaction within family ( } 40 \text { different } \\
\text { texts) }\end{array}$ & $\begin{array}{l}\text { a. interaction among family members } \\
\text { (26) } \\
\text { b. parenting skills (i.e. parent's men- } \\
\text { tal health, skills to transfer abili- } \\
\text { ties to another environments) (13) } \\
\text { c. attachment (4) } \\
\text { d. positive image (3) } \\
\text { e. attunement (1) }\end{array}$ & $\begin{array}{l}\text { a. Abad, 2007; Abad \& Williams, 2006; } \\
\text { Allgood, 2005; Bargiel, 2004; Bunt, } \\
\text { 2002; Drake 2008; Drake, 2011; Dun, } \\
\text { 1995; Gilboa \& Roginsky, 2010; Hi- } \\
\text { bben, 1992; Jonsdottir, 2002; Kelly, } \\
\text { 2011; Ledger, 2011; Lindenfelser et } \\
\text { al., 2012; McKenzie \& Hamlett, } \\
\text { 2005; Nall \& Everitt, 2005; } \\
\text { O'Callaghan \& Jordan, 2011; Old- } \\
\text { field, 1993; Oldfield et al., 2003; } \\
\text { Pasiali, 2012b; Shoemark, 1996; } \\
\text { Shoemark, 2004; Shoemark, 2011; } \\
\text { Thompson, 2012; Trolldalen, 1997; } \\
\text { Woodward, 2004 } \\
\text { b. Abad \& Williams, 2006; Ayson, } \\
\text { 2008; Cunningham, 2011; Dun, 1995; } \\
\text { Edwards et al., 2007; Horvat \& } \\
\text { O'Neill, 2008; Muller \& Warwick, } \\
\text { 1997; Nicholson et al., 2008; Nichol- } \\
\text { son et al., 2010; Oldfield, 2006; } \\
\text { Voigt, 2003; Walworth, 2009; } \\
\text { Williams et al., 2012 } \\
\text { c. Bargiel, 2004; Cunningham, 2011; } \\
\text { Kelly, 2011; O'Callaghan \& Jordan, } \\
\text { 2011 } \\
\text { d. Muller \& Warwick, 1997; Oldfield, } \\
\text { 2011 Thompson, 2012 } \\
\text { e. Levinge, 2011 }\end{array}$ \\
\hline Interaction (29 different texts) & $\begin{array}{l}\text { a. engagement (gaining attention, eye } \\
\text { contact) (12) } \\
\text { b. increased intentions and initiatives } \\
\text { (choice making, active role, re- } \\
\text { sponse time) (11) } \\
\text { c. positive interaction (9) } \\
\text { d. turn-taking and reciprocity (6) } \\
\text { e. sustained attention ( } 4\end{array}$ & $\begin{array}{l}\text { a. Bruce \& High, 2012; Bunt, 2002; } \\
\text { Finnigan \& Starr, 2010; Hibben, } \\
\text { 1992; Kim et al., 2009; Lecourt, 1991; } \\
\text { Oldfield, 1991; Oldfield, 2006; } \\
\text { Pasiali, 2012b; Robb, 2000; Salas \& } \\
\text { Gonzales, 1991; Tomlinson, } 2010 \\
\text { b. Aasgaard, 2001; Bruce \& Brown, } \\
\text { 2012; Dun, 1999; Elefant \& Wigram, } \\
\text { 2005; Guerrero \& Turry, 2012; Loth, } \\
\text { 2008; Register, 2004; Muller \& War- } \\
\text { wick, 1997; Robb, 2000; Standley \& } \\
\text { Hughes, 1996; Williams, 2012 } \\
\text { c. Bunt, 2002; Finnigan \& Starr, 2010; } \\
\text { Guerrero \& Turry, 2012; Kim et al., } \\
\text { 2008; Loth, 2008; Muller \& Warwick, } \\
\text { 1997; Pasiali, 2012b; Perry, 2003; } \\
\text { Skewes \& Thompson, 1998 } \\
\text { d. Davies \& Rosscornes, 2012; Drake, } \\
\text { T. 2008; Dun, 1999; Levinge, 2011; } \\
\text { Perry, 2003; Robb, 2000 }\end{array}$ \\
\hline
\end{tabular}




\begin{tabular}{|c|c|c|}
\hline Social areas & Definition & Reference \\
\hline & & $\begin{array}{l}\text { e. Lecourt, 1991; Perry, 2003; Sussman, } \\
\text { 2009; Tomlinson, } 2010\end{array}$ \\
\hline Communication (29 different texts) & $\begin{array}{l}\text { a. enabling and enhancing communi- } \\
\text { cation (14) } \\
\text { b. medium for communication (vocal- } \\
\text { ization, verbalization) (11) } \\
\text { c. non-verbal communication (5) }\end{array}$ & $\begin{array}{l}\text { a. Dun, 1999; Hall, 2012; Hibben, 1992; } \\
\text { Lindenfelser et al., 2008; Loth, } \\
\text { 2008; Nicholson et al., 2008; Old- } \\
\text { field, 1991; Pasiali, 2012b; Perry, } \\
\text { 2003; Salas \& Gonzales, 1991; Shoe- } \\
\text { mark, 1999; Skewes \& Thompson, } \\
\text { 1998; Trevarthen et al., 1998; Wood- } \\
\text { ward, } 2004 \\
\text { b. Aasgaard, 2005; Beathard \& Krout, } \\
\text { 2008; Berger, 2002; Bunt 2002; } \\
\text { Gilboa \& Roginsky, 2010; Jones \& } \\
\text { Oldfield, 1999; Kennelly et al., 2001; } \\
\text { Levinge, 1990; Oldfield, 2006; Robb, } \\
\text { 2000; Tomlinson, 2010 } \\
\text { c. Dun, 1995; Kim et al., 2008; Levinge, } \\
\text { 2011; Oldfield, 2011; Skewes \& } \\
\text { Thompson, 1998 }\end{array}$ \\
\hline Social behavior (9 different texts) & $\begin{array}{l}\text { a. decreased problem behavior (3) } \\
\text { b. concentration ( } 3 \text { ) } \\
\text { c. need of control reduced (1) } \\
\text { d. decreased stereotypic behaviour } \\
\text { (1) } \\
\text { e. acceptance of differences (1) }\end{array}$ & $\begin{array}{l}\text { a. de Mers et al., 2009; Oldfield, 1991; } \\
\text { Register \& Humpal, } 2007 \text { (3) } \\
\text { b. Loth, 2008; Oldfield et al., 2003; } \\
\text { Robb, } 2003 \\
\text { c. Brown, } 2002 \\
\text { d. Muller et al., } 1997 \\
\text { e. Skewes \& Thompson, } 1998\end{array}$ \\
\hline
\end{tabular}

tences were in the minority (Register, 2001; Standley \& Hughes, 1997) as well as pain relief (Edwards, 1994; Sweeney-Brown, 2005).

These are the effects of early childhood music therapy, which did not fit into previous categories.

Assessment is an important part of therapy when setting goals for a process. There were five papers referring to this matter within this age group. Focus on this material was on experiences where music therapy could have provided some kind of information, which was not revealed through other assessment tools.

A very different kind of approach was introduced in two papers where the cost-effectiveness was the focus (Loewy et al., 2005; Walworth, 2005). Both researches examined the cost-effectiveness of music therapy in the pediatric healthcare setting aiming to reduce the amount of sedation for patients undergoing various procedures. This kind of research is rare within the music therapy field but might be one-direction researchers will be encouraged to take in the future.

\section{Specific findings}

In this section, the authors reflect on several aspects, which they found particularly interesting. This section is mainly descriptive rather than analytical and it is not part of the more structured analysis. 
Table 4. Emotional areas

\begin{tabular}{|c|c|c|}
\hline Emotional areas & Definition & References \\
\hline Positive factors (36 different texts) & $\begin{array}{l}\text { a. fun, joy, enjoyment, playfulness } \\
(20) \\
\text { b. atmosphere and positive attitude } \\
\text { ( } 7 \text { ) } \\
\text { c. new insights, memories (9) } \\
\text { d. motivation (8) } \\
\text { e. normalization and reduced symp- } \\
\text { toms (3) }\end{array}$ & $\begin{array}{l}\text { a. Aasgaard 2005; Ayson, 2008; Bar- } \\
\text { rera \& Rykov, 2002; Bruce \& High, } \\
\text { 2012; Drake, 2008; Dun, 1999; Dun, } \\
\text { 2007; Gfeller, 1990; Hadley, 1996; } \\
\text { Hall, 2012; Hendon \& Bohon, 2008; } \\
\text { Kim et al., 2009; Lecourt, 1991; Lin- } \\
\text { denfelser et al. 2012; Loth, 2008; } \\
\text { Oldfield, 1991; Oldfield, 1993; Old- } \\
\text { field, 2011; Shoemark \& Dearn, } \\
\text { 2008; Thompson, 2012 } \\
\text { b. Dun, 1999; Dun, 2007; Edwards, } \\
\text { 1994; Pasiali, 2012b; Schwartz, 2011; } \\
\text { Shoemark, 1999; Shoemark, 2004 } \\
\text { c. Allgood, 2005; Darnley-Smith, 2003; } \\
\text { Dun, 2007; Lindenfelser et al., 2008; } \\
\text { Nall \& Everitt, 2005; O'Callaghan \& } \\
\text { Jordan, 2011; Rees, 2005; Schwartz, } \\
\text { 2011; Shoemark, 2004 } \\
\text { d. Dun, 1995; Elefant \& Wigram 2005; } \\
\text { Finnigan \& Starr, 2010; Gfeller, 1990; } \\
\text { Kim et al., 2009; Oldfield, 1991; } \\
\text { Skewes \& Thompson, 2009; Tomlin- } \\
\text { son, 2010 } \\
\text { e. Ayson, 2008; Dun, 2007; Gold et al., } \\
\text { 2001 }\end{array}$ \\
\hline Supportive factors (33 different texts) & $\begin{array}{l}\text { a. emotional support and - sharing, } \\
\text { nurture and comfort, soothing (17) } \\
\text { b. feeling of mastery ( } 6 \text { ) } \\
\text { c. resources and resilience (6) } \\
\text { d. self confidence (5) } \\
\text { e. coping skills ( } 4 \text { ) }\end{array}$ & $\begin{array}{l}\text { a. Ayson, 2008; Barrera \& Rykov 2002; } \\
\text { Burke 1991; Cunningham, 2011; } \\
\text { Davis, G. 2005; Drake, 2011; Dun, } \\
\text { 1995; Dun, 1999; Dun, 2007; Jons- } \\
\text { dottir, 2002; Levinge, 2011; Linden- } \\
\text { felser et al., 2012; O'Callaghan \& } \\
\text { Jordan, 2011; Salas \& Gonzales, } \\
\text { 1991; Shoemark, 1999; Sweeney- } \\
\text { Brown, 2005; Trevarthen et al., } 1998 \\
\text { b. Beer, 1990; Bruce \& High, 2012; Ed- } \\
\text { wards, 1994; Hadley, 1996; Oldfield, } \\
\text { 1991; Trolldalen, 1997 } \\
\text { c. Darnley-Smith, 2003; Dun, 1995; } \\
\text { Dun, 1999; Irgens-Moller, 1999; } \\
\text { O'Callaghan \& Jordan, 2011; Shoe- } \\
\text { mark, 2004 } \\
\text { d. Aasgaard 2005; Davies \& Ross- } \\
\text { cornes, 2012; Hall, 2012; Ledger, } \\
\text { 2011; Woodward, 2004 } \\
\text { e. Beer, 1990; Berger, 2002; Hadley, } \\
\text { 1996; Shoemark, 2006 }\end{array}$ \\
\hline $\begin{array}{l}\text { Expressive and regulating qualities ( } 27 \\
\text { different texts) }\end{array}$ & a. expressing emotions, creativity (17) & $\begin{array}{l}\text { a. Aasgaard, 2001; Aasgaard, 2005; } \\
\text { Beer, 1990; Brackley 2012; Bunt, }\end{array}$ \\
\hline
\end{tabular}




\begin{tabular}{|c|c|c|}
\hline Emotional areas & Definition & References \\
\hline & $\begin{array}{l}\text { b. regulation of emotions, calming } \\
\text { and activating (13) }\end{array}$ & $\begin{array}{l}\text { 2002; Burke, 1991; Dun, 1995; Guer- } \\
\text { rero \& Turry, 2012; Hong et al., 1998; } \\
\text { Horvat \& O Neill, 2008; Irgens- } \\
\text { Moller, 1999; Lecourt, 1991; Levinge, } \\
\text { 2011; Tomlinson, 2010; Trevarthen } \\
\text { et al., 1998; Salas \& Gonzales, 1991; } \\
\text { Warnock, } 2011 \\
\text { b. Aldridge, 1993; Ayson, 2008; Bargiel, } \\
\text { 2004; Barrera \& Rykov, 2002; Berg- } \\
\text { er, 2002; Brackley, 2012; Hadley, } \\
\text { 1996; Hong et al., 1998; Lecourt, } \\
\text { 1991; Loewy, 2004; Lindenfelser et } \\
\text { al., 2004; Lindenfelser et al., 2012; } \\
\text { Pasiali, 2012b }\end{array}$ \\
\hline Other factors ( 13 different texts) & $\begin{array}{l}\text { a. development of self, separation, } \\
\text { and personal development (11) } \\
\text { b. coherence (structuring and catego- } \\
\text { rizing) (2) } \\
\text { c. emotional synchronicity (1) }\end{array}$ & $\begin{array}{l}\text { a. Beer, 1990; Brown, 2002; Cunning- } \\
\text { ham, 2011; Darnley-Smith, 2003; } \\
\text { Horvat \& O' Neill, 2008; Levinge, } \\
\text { 1990; Shoemark, 2011; Trevarthen } \\
\text { et al., 1998; Trolldalen, 1997; Salas } \\
\text { \& Gonzales, 1991; Warnock, } 2011 \\
\text { b. Lecourt, 1991; Salas \& Gonzales, } \\
1991 \\
\text { c. Kim et al., } 2009\end{array}$ \\
\hline
\end{tabular}

Table 5. Physical and cognitive areas

\begin{tabular}{|c|c|c|}
\hline Physical / cognitive areas & Definition & References \\
\hline $\begin{array}{l}\text { Physical and motor areas (14 different } \\
\text { texts) }\end{array}$ & $\begin{array}{l}\text { a. relaxation (5) } \\
\text { b. motoric abilities ( } 4 \text { ) } \\
\text { c. pain relief ( } 2 \text { ) } \\
\text { d. neurological development (1) } \\
\text { e. eating behavior (1) } \\
\text { f. linking sensory perceptions ( } 1 \text { ) }\end{array}$ & $\begin{array}{l}\text { a. Aldridge 1993; Loewy, 2004; Loewy } \\
\text { et al., 2005; Oldfield, 1991; Wal- } \\
\text { worth, 2005 } \\
\text { b. Beathard \& Krout, 2008; Bruce \& } \\
\text { High, 2012; Duffy \& Fuller, 2000; } \\
\text { Wylie 1996 } \\
\text { c. Edwards, 1994; Sweeney-Brown, } \\
2005 \\
\text { d. Shoemark, } 2004 \\
\text { e. Dellatan, } 2003 \\
\text { f. Lecourt, } 1991\end{array}$ \\
\hline Cognitive areas (11 different texts) & $\begin{array}{l}\text { a. language development (5) } \\
\text { b. competences, ability to learn ( } 4 \text { ) } \\
\text { c. prewriting and - print concepts ( } 2 \text { ) } \\
\text { d. reading competences (1) } \\
\text { e. awareness (1) }\end{array}$ & $\begin{array}{l}\text { a. Kennelly et al., 2001; Lim, 2012; Lim } \\
\text { \& Draper, 2011; Gross \& Linden, } \\
\text { 2010; Guerrero \& Turry, } 2012 \\
\text { b. Elefant \& Wigram, 2005; Gold et al., } \\
\text { 2001; Gross \& Linden, 2010; } \\
\text { Humpal, } 2012 \\
\text { c. Register 2001; Standley, } 1997 \\
\text { d. Register, } 2001 \\
\text { e. Dun, } 1999\end{array}$ \\
\hline
\end{tabular}


Table 6. Other areas

\begin{tabular}{|c|c|c|}
\hline Other areas & Definition & References \\
\hline Assessment (5 different texts) & $\begin{array}{l}\text { a. Tool for assessment ( } 3 \text { ) } \\
\text { b. New information about child (2) }\end{array}$ & $\begin{array}{l}\text { a. Hadley, 1996; Layman et al., 2002; } \\
\text { O'Neill, } 2012 \\
\text { b. Irgens-Moller, 1999; Wigram, } 1995\end{array}$ \\
\hline Other dimensions (4) & $\begin{array}{l}\text { a. Spirituality (2) } \\
\text { b. holistic view (1) } \\
\text { c. economical viewpoint (1) }\end{array}$ & $\begin{array}{l}\text { a. Sweeney, 2003; Sweeney-Brown, } \\
2005 \\
\text { b. Shoemark \& Dearn, } 2008 \\
\text { c. Loewy et al., 2005; Walworth, } 2005\end{array}$ \\
\hline
\end{tabular}

\section{Books}

Music Therapy in Children's Hospices (Pavlicevic, 2005) is currently the only book in the field of music therapy in palliative care with children. Music therapy with children and their families (Oldfield \& Flower, 2008) has been an important opening for future development of family work. The same applies to Edwards' book (2011), which focuses on the use of music therapy in promoting attachment across community, medical, and school based contexts. Music therapy in schools - working with children of all ages in mainstream and special education (Tomplinson et al., 2012) is an opening to the school world where small children and their carers are taken into consideration. Early childhood music therapy and autism spectrum disorders (Kern \& Humpal, 2012) is relevant for this research because of its specific topic.

\section{Large number of participants}

There were several papers, which included a large amount of research participants, defined for the purposes of this review as 50 or more cases. In music therapy research in general, large numbers are the exception rather than the rule. In this study, there seem to be several papers with a large number of participants. This was particularly evident in the Sing\&Grow reports (Abad, 2007; Abad \& Williams, 2006; Nicholson et al., 2010; Nicholson et al., 2008; Williams et al., 2012). Participants numbers were 850 (Nicholsson et al., 2010) and 635 families (Abad, 2007) per paper. Other program or curriculum based interventions also seemed to have numerous participants. A preventive intervention The Music Together Program (MacKenzie \& Hamlett, 2005) gathered information from 140 families. Another preventive program offered in schools was with 80 dyads participating a family-based music therapy program (Kelly, 2011). Eighty-six kindergarten children participated in a music therapy program designed to teach reading skills (Register, 2004) and 50 children participated in a research project where music therapy was designed to enhance prereading and writing skills (Register, 2001).

Previously introduced researches into cost-effectiveness (Loewy et al., 2005; Walworth, 2005) also included many participants. Walworth's study included 166 patients between 6 months and 13 years and Loewy's 60 patients from 1 month through 5 years of age. A pilot study explored the effectiveness of interactive music therapy of hospitalized children with cancer (Barrera et al., 2002). Sixty-five children participated, of which 33 were 0-5 years of age. Seventy infants under 2 years and their parents were examined regarding responsiveness and infant social development (Walworth, 2009). Hospitalized children's mood was tested in an investigation where 60 children from 13 months to 12 years participated (Hendon \& Bohon, 2007).

Typically, the papers that included a large number of participants seemed to describe quite a structured way of working. These papers often evaluated programs and curriculums, usually with groups. Music therapy was often a short-term intervention. Different questionnaires were often used as well as observational notes. This was un- 
derstandable and often necessary given the large amount of data involved. However, this means that the data can often not be analysed in depth and in many cases the why questions remain unresolved. Generalizations can be made but specific information might be lacking. The meaning of such papers is important in situations where the benefits of music therapy need to become visible and convince the policymakers.

\section{Length of music therapy processes}

The sources revealed that early childhood music therapy is commonly accomplished as a short-term intervention with few services being provided for longer than a year. Only 10 papers were identified which mentioned that music therapy lasted at least a year. However, this information was not available in every paper.

The longest therapy processes mentioned lasted 4 or 5 years (Horvat \& O’Neill, 2008; Warnock, 2011). Mostly longer work lasted 1 to 2 years (Brown, 2002; Bunt, 2002; Dun, 2007; Hong et al., 1998; Lecourt, 1991; Levinge, 1990; Oldfield, 2006; Shoemark, 1996; Trevarthen et al., 1998). Interestingly all of these papers were written before 2008. This result might be linked to the previous finding, which showed that individual music therapy was dominant at the same time. All the long-term work was individual or dyadic/family work.

\section{Discussion}

The main aim of this paper was to conduct an overview of the early childhood music therapy literature. The authors wanted to get a picture of the field worldwide an idea of groupings within the subject area and find possible trends but also gaps in literature. In addition, the target was to conceptualize the field, provide a context, and define a history of what has been done.

\section{Reflection on most important findings}

Based on the data analysis (both overall analysis and the analysis of the research data) the results showed that older children were more often written about. Once again based on the assumption that the amount of literature reflects clinical trends, the authors suggest that older children might be more likely to receive music therapy treatment than younger children. Although the exclusion of music therapy in neonatal care and with premature infants has affected the results. Also, it is possible that children under 2 years are not so often referred to music therapy because of the lack of necessary evaluation and diagnosis in such a young age.

According to the results, including the carer in therapy during the first year was almost as common as it was with 5-year-old children. Different types of therapy were quite the same in children with 3 years of age and individual music therapy was the most common therapy type with 5-year-old children.

Historically it seems that during the last 22 years there have been changes not only in amount of texts but also on the types of therapy presented. The dominance from individual work has been shifting to family/dyadic work. Conclusions might be that music therapy has grown as a profession and publishing both research and clinical material has increased. Also, there is a possibility that the interest in clinical practice has been changing somewhat in favour of small children.

The edited books undoubtedly affected the results. There were three books published in 2011-2012 (Edwards, 2011; Kern \& Humpal, 2012; Tomlinson et al., 2012) of which 18 chapters matched the criteria for this research. The dominance of individual work between 2001 and 2005 cannot clearly be explained. Again there were edited books (Bunt \& Hoskyns, 2002; Pavlicevic, 2005) affecting the data but not so obviously as mentioned earlier. It could be that during those years the awareness of music therapy with small children increased, resulting in more writing on the subject.

Some of the differences in types of therapy changing across the targeted time span could be related to legal and regulatory options for service for young children. In the 
United States, focus for treatment moved significantly from individual, clinic based work to family and group based work due to changing emphasis under the Individual with Disabilities Education Act (IDEA). In Australia, the funding for Sing\&Grow could be seen as a driving force in the changing types of practice.

Looking at types of intervention, active methods were most common with singing and playing with instruments the most prevalent. Only 66 texts (from 116 texts) reported using improvisation. This could be partly because the texts did not articulate details like this clearly enough. Also, music therapy programs or group work (Aldridge, 1993; Edwards et al., 2007; Nicholson et al., 2010), which might be well structured and planned in advance seemed not to use improvisation as much as other kinds of approaches. In addition, strict research design might have limited the use of different methods (i.e. Elefant \& Wigram, 2005; Finnigan \& Starr, 2010; Lim \& Draper, 2011).

Despite the difficulties of categorizing client groups, it seemed that children with ASD were most strongly represented together with paediatric patients and children with developmental disabilities. Preventive music therapy approaches were well represented, whereas the services for children with mental disorders and children with communication disorders were smaller in number.

This research could not evaluate different music therapy approaches in a systematic way because many writers did not name or describe their exact approach. However, when looking at those authors' texts who mentioned some aspects, it would appear that psychodynamic approaches were in the minority and the focus when carrying out early childhood music therapy was usually on creative and improvisational music therapy. This could explain why the concepts of reflective function, mentalization, and parent's representations were absent, even though these issues are currently an important focus for early interaction research worldwide (Fonagy, 2012; Pajulo et al., 2012; Philipp, 2012; Solbakken et al., 2011).

When analysing the data from the viewpoint of reported effects of music therapy (see tables $3,4,5,6$ ), authors mentioned positive effects in four different areas: a) social areas, b) emotional areas, c) physical and academic skill areas, and d) other areas. Motor skills were emphasized especially with children with developmental delays, though only in a few papers, and only one text took a neurological viewpoint.

Interactions among family members were emphasized, and parenting skills were also quite well represented. Increased intentions and initiatives, engagement as well as turn-taking and reciprocity emerged quite strongly from the data. It was somewhat surprising that non-verbal interaction was only mentioned in a few texts. The authors' assumption had been that within the population of 0 to 5-year-old children it would have been more emphasized. According to this data it also appeared that countertransference was not commonly mentioned or focused on in the texts.

Decreased problem behavior, decreased stereotypic behaviour, and acceptance of differences, was in the minority when examining the effects of music therapy. One could assume that problems within this area are not so big in this age group. Also, it might be a matter of research design and targets, as research designs might not often identify measures where decreases of behavior could be detected.

Early childhood music therapy literature emphasized the positive factors. The importance of fun and enjoyment was underlined throughout all client groups. Resilience is currently a subject, which is frequently mentioned in the literature concerning children and families (Papousek, 2011; Pasiali, 2012a; Pearce, 2011; Sawyer \& Burton, 2012). Both from the viewpoints of humanity and economy, it seems it would be beneficial to focus on the strengths of clients. In this data, six articles (Darnley-Smith \& Patey, 2003; Dun, 1995; Dun, 1999; Irgens-Moller, 1999; O’Callaghan \& Jordan, 2011; Shoemark, 2004) wrote about resources and resilience as well as about feelings of mastery.

Specific features of the research data were investigated only in high impact research publications. The freer the framework of writing was, the more general were the conclusions. 


\section{Limitations}

Collecting data with such a broad inclusion criteria was very demanding and constituted a limitation to this investigation. Despite the systematic approach, it is still possible there are papers which met the criteria for inclusion but were not included in the research. On the other hand, the quite strict definition of music therapy limited the data and left some research papers out. However, the amount of data was substantive and it is reasonable to assume that single additional texts would not have impacted the overall results.

The quality of the research also suffered because of the large amount of texts. The controlled experiments and quantifiable data were not in focus, and the "critical reading" (Aveyard, 2010; Randolph 2009) was not carried out in this research. Because of the large amount of texts, the analysis was carried out more superficially than with a strictly selected, small amount of data. The qualitative data in particular, could have been analysed in a much more detailed and deeper way if the data had been less extensive.

The categorizing in this research has its limits because of overlaps between different categories (i.e. group work could be either for individual children but also for dyads or families). Also, geographical divergence of the terms and the classification regarding the clinical population has had an influence on the analysis and results. Though an objective standpoint was tried to be maintained, the authors' own backgrounds, experiences, and standpoints have with some extent influenced the interpretations. The research would look different if the authors would have been different.

Despite the limitations of this research, the authors believe it gives an overall idea of early childhood music therapy practice and how this praxis is accomplished. It gives us guidelines of early childhood music therapy practice and a means of accomplishing it. It gives us guidelines of what kind of client groups early childhood music therapy takes place with and what the effects of this intervention are seen to be. We also gain an understanding of gaps in the literature, what is missing, and which area it would be beneficial to consider in more depth.

\section{Guidelines for future}

There are some issues for the future, which should be pointed out. From the researcher's perspective it would be important that articles, both clinical and research articles, included precise information about a) the age of the target group (for example: "preschool"/"kindergarten student"/"school age" referring to different age groups in different countries), b) the definition of the intervention (for example: education or therapy), c) type of work (who was present in sessions), d) duration and frequency of therapy (how many times per week, how long the sessions lasted, how many sessions all together), e) methods used. In addition, the framework of therapy and the therapist (i.e. psychotherapeutic, creative or improvisational music therapy) would be important to define. All this information should be easy to find and the structure of the article should be logical. Ideally this information should be available in the abstract of the article.

Within the context of early childhood music therapy, children from birth to 2 years of age seemed to be in the minority. Research in the fields of communication disorders and psychiatric care were low incidence and hospice care, sensory impairment, and parenting issues minimal. The research from field of attachment was missing completely. The authors suggest these areas to be researched more and written about in future. Long-term processes were in a minority and deeper information was often lacking. It was decided, for the sake of simplicity, not to distinguish between group work for individual children and group work with families. In retrospect, this might have been useful to look at in more detail and is a recommendation for future research.

More specific information is needed. In this data, the interactional and the emotional areas were described in a general way (i.e. music therapy helps, supports, and creates interaction), but more exact information was mostly not forthcoming. The pro- 
fession needs more detailed knowledge, which is something for future researchers to consider. In addition, related areas of research, cross-scientific viewpoints and common fields of interests could be taken into consideration. The current world effectiveness should also be examined as well as the effects of music therapy in everyday life.

Early childhood music therapy is a multifaceted field with different client groups, types of work, approaches, and areas of emphasis. General trends have been determined, maybe it is now time to investigate deeper and in a more focused way considering our clients, our profession, and our funders.

\section{Funding}

The Finnish Cultural Foundation under Grant number 00120959 and Finnish Cultural Foundation's Häme Regional Fund under Grants 15091874 and 15141655 supported this work.

\section{References}

Aasgaard, T. (2005). Song creations by children with cancer - process and meaning. In D. Aldridge (Ed.), Case study designs in music therapy (pp. 67-96). London, Philadelphia: Jessica Kingsley Publishers.

Aasgaard, T. (2001). An ecology of love: aspects of music therapy in the pediatric oncology environment. Journal of Palliative Care, 17(3), 177-224.

Abad, V. (2007). Early intervention music therapy: Reporting on a 3-year project to address needs with at-risk families. Music Therapy Perspectives, 25, 52-58, https://doi.org/10.1093/ $\mathrm{mtp} / 25.1 .52$.

Abad, V., \& Williams, K. (2006). Early intervention music therapy for adolecsent mothers and their children. British Journal of Music Therapy, 20(1), 31-38, https://doi.org/10.1177/ 135945750602000106.

Achenbach, C. (2012). Nordoff-Robbins music therapy in a nursery setting. Supporting music therapy students on placement. In J. Tomlinson, P. Derrington, \& A. Oldfield (Eds.), Music therapy in schools. Working with children of all ages in mainstream and special education (pp. 47-59). London, Philadelphia: Jessica Kingsley Publishers.

Aldridge, K. (1993). The use of music to relieve pre-operational anxiety in children attending day surgery. Australian Journal of Music Therapy, 4, 19-35.

Allgood, N. (2005). Parents' perceptions of family-based group music therapy for children with autism spectrum disorders. Music Therapy Perspectives, 23, 92-99, https://doi.org/ 10.1093/mtp/23.2.92.

Aveyard, H. (2010). Doing a literature review in health and social care: A practical quide (2nd ed.). Berkshire, GBR: Open University Press.

Ayson, C. (2008). Child-parent wellbeing in a paediatric ward. The role of music therapy in supporting children and their parents facing the challenge of hospitalisation. Voices: $A$ World Forum for Music Therapy, 8(1), https://doi.org/10.15845/voices.v8i1.449.

Bargiel, M. (2004). Lullabies and play songs. Theoretical considerations for an early attachment music therapy intervention through parental singing for developmentally at-risk infants. Voices: A World Forum for Music Therapy, 4(1), https://doi.org/10.15845/ voices.v4i1.149.

Barrera, M. E., Rykov, M. H., \& Doyle, S. L. (2002). The effects of interactive music therapy on hospitalized children with cancer: A pilot study. Psycho-Oncology, 11(5), 379-388, https://doi.org/10.1002/pon.589.

Bartram, P. (1991). Improvisation and play in the therapeutic engagement of a five-year-old boy with physical and interpersonal problems. In K. Bruscia (Ed.), Case studies in music therapy (2nd ed., pp. 137-152). Gilsum, NH: Barcelona Publishers.

Beathard, B. (2008). A music therapy clinical case study of a girl with childhood apraxia of speech: finding Lily's voice. The Arts in Psychotherapy, 35(2), 107-116, https://doi.org/ 10.1016/j.aip.2008.01.004. 
Beer, L. E. (1990). Music therapy: sounding your myth. Music Therapy, 9(1), 35-43, https://doi.org/10.1093/mt/9.1.35.

Berger, D. S. (2002). Music therapy, sensory integration and the autistic child. London, Philadelphia: Jessica Kingsley Publishers.

Brackley, J. (2012). Music therapy and the expression of anger and aggression. Working with aggressive behavior in children aged five to nine who risk mainstream school exclusion. In J. Tomlinson, P. Derrington, \& A. Oldfield (Eds.), Music Therapy in schools. Working with children of all ages in mainstream and special education (pp. 89-102). London, Philadelphia: Jessica Kingsley Publishers.

Brown, S. (2002). "Hullo object! I destroyed you!”. In L. Bunt \& S. Hoskyns (Eds.), The handbook of music therapy (pp. 84-96). New York: Brunner-Routledge.

Bruce, A., \& High, S. (2012). Multiple views of music therapy. In J. Tomlinson, P. Derrington, \& A. Oldfield (Eds.), Music therapy in schools. Working with children of all ages in mainstream and special education (pp. 71-83). London, Philadelphia: Jessica Kingsley Publishers.

Bruscia, K. (1998). Defining music therapy (2nd ed.). London: Routledge.

Bunt, L. (2002). Suzanna's story: music therapy with a pre-school child. In L. Bunt \& S. Hoskyns (Eds.), The handbook of music therapy (pp. 71-83). New York: Brunner-Routledge.

Bunt, L., \& Hoskyns, S. (Eds.). (2002). The handbook of music therapy. New York: BrunnerRoutledge.

Burke, K. (1991). Music therapy in working through a preschooler's grief: expressing rage and confusion. In K. Bruscia (Ed.), Case studies in music therapy (2nd ed., pp. 127-135). Gilsum, NH: Barcelona Publishers.

Carpente, J. (2012). DIR/Floortime model. Introduction and considerations for improvisational music therapy. In P. Kern \& M. Humpal (Eds.), Early childhood music therapy and autism spectrum disorders. Developing potential in young children and their families (pp. 145-161). London, Philadelphia: Jessica Kingsley Publishers.

Cassidy, J. W., \& Ditty, K. M. (2001). Gender differences among newborns on a transient otoacoustic emissions test for hearing. Journal of Music Therapy, 38(1), 28-35, https://doi.org/10.1093/jmt/38.1.28.

Cassity, M. D., \& Cassity, J. E. (2006). Multimodal psychiatric music therapy for adults, adolescents, and children. A clinical manual (3rd ed.). London, Philadelphia: Jessica Kingsley Publishers.

Cmap. (n.d.). Retrieved from http://cmap.ihmc.us

Cunningham, J. (2011). Music therapy to promote attachment between mother and baby in marginalized communities. In J. Edwards (Ed.), Music therapy and parent-infant bonding (pp. 115-126). Oxford: Oxford University press.

Darnley-Smith, R., \& Patey, H. M. (2003). Music therapy. London: Sage Publications.

Davies, E., \& Rosscornes, C. (2012). Setting up and developing music therapy at a children's centre, for pre-school children and their families and carers. In J. Tomplinson, P. Derrington, \& A. Oldfield (Eds.), Music therapy in schools. Working with children of all ages in mainstream and special education (pp. 19-32). London, Philadelphia: Jessica Kingsley Publishers.

Davis, G. (2005). Living community: Music therapy with children and adults in a hospice setting. In M. Pavlicevic (Ed.), Music therapy in children's hospices: Jessie's Fund in action (pp. 124-138). London, Philadelphia: Jessica Kingsley Publishers.

Dellatan, A. K. (2003). The use of music with chronic food refusal: A case study. Music Therapy Perspectives, 21, 105-109, https://doi.org/10.1093/mtp/21.2.105.

DeMers, C. L., Tincani, M., van, R. K., \& Higgins, K. (2009). Effects of music therapy on young children's challenging behaviors: A case study. Music Threrapy Perspectives, 27, 88-96, https://doi.org/10.1093/mtp/27.2.88. 
Dileo, C. (2005). Reviewing the literature. In B. Wheeler (Ed.), Research in music therapy quantitative and qualitative perspectives (2nd ed., pp. 105-111). Gilsum, NH: Barcelona Publishers.

Drake, T. (2011). Becoming in tune: The use of music therapy to assist the developing bond between traumatized children and their new adoptive parents. In J. Edwards (Ed.), Music therapy and parent-infant bonding (pp. 101-114). Oxford: Oxford University press.

Drake, T. (2008). Back to basics. Community-based music therapy for vulnerable young children and their parents. In A. Oldfield \& C. Flower (Eds.), Music therapy with children and their families (pp. 37-51). London, Philadelphia: Jessica Kingsley Publishers.

Duffy, B., \& Fuller, R. (2000). Role of music therapy in social skills development in children with moderate intellectual disability. Journal of Applied Research in Intellectual Disabilities, 13(2), 77-89, https://doi.org/10.1046/j.1468-3148.2000.00011.x.

Dun, B. (1999). Creativity and communication. Aspects of music therapy in a children's hospital. In D. Aldridge (Ed.), Music therapy in palliative care. New voices (pp. 59-67). London, Philadelphia: Jessica Kingsley Publishers.

Dun, B. (1995). A different beat: Music therapy in children's cardiac care. Music Therapy Perspectives, 13, 36-44.

Dun, B. (2007). Journeying with Olivia: Bricolage as a framework for understanding music therapy in paediatric oncology. Voices: A World Forum for Music Therapy, 7(1), https://doi.org/10.15845/voices.v7i1.464.

Edwards, J. (2011). Music therapy and parent-infant bonding. Oxford: Oxford University press.

Edwards, J., Scahill, M., \& Phelan, H. (2007). Music therapy: Promoting healthy mother-infant relations in the vulnerable refugee and asylym seeker community. In J. Edwards (Ed.), Music therapy: Promoting health and creating community in healthcare (pp. 154-168). Cambridge: Cambridge Scholars Publishing.

Edwards, J. (1994). The use of music therapy to assist children who have severe burns. Australian Journal of Music Therapy, 5(1), 3-6.

Edwards, J., \& Kennelly, J. (2004). Music therapy in paediatric rehabilitation. Nordic Journal of Music Therapy, 13, 112-126, https://doi.org/10.1080/08098130409478108.

Egger, H. (2009). Toddler with temper tantrums. A careful assessment of a dysregulated preschool child. In C. A. Galanter \& P. S. Jensen (Eds.), DSM-IV-TR casebook for child mental health (pp. 365-384). Arlington: American psychiatric publishing, Ink.

Elefant, C. (2010). Unmasking hidden resources. Communication in children with severe developmental disabilities in music therapy. In V. Karkou (Ed.), Arts Therapies in Schools (pp. 243-258). London, Philadelphia: Jessica Kingsley Publishers.

Elefant, C., \& Wigram, T. (2005). Learning ability in children with Rett syndrome. Brain \& Development, 27, 97-101, https://doi.org/10.1016/j.braindev.2005.03.020.

English Oxford living dictionaries. (n.d.). Retrieved from http://en.oxforddictionaries.com

Finnigan, E., \& Starr, E. (2010). Increasing social responsiveness in a child with autism. A comparison of music and non-music interventions. Autism, 14(4), 321-348, https://doi.org/ $10.1177 / 1362361309357747$.

Fonagy, P. (2012). Handbook of mentalizing in mental health practice. Arlington, VA: American psychiatric publishing, Inc.

Galvan, J. L. (2009). Writing literature reviews. A guide for students of the social and behavioral sciences (4th ed.). Glendale, CA: Pyrczak Publishing.

Gfeller, K. (1990). A cognitive-linguistic approach to language development for the preschool child with hearing impariment: implications for music therapy practice. Music Therapy Perspectives, 8, 47-51, https://doi.org/10.1093/mtp/8.1.47.

Gilboa, A., \& Roginsky, E. (2010). Examining the dyadic music therapy treatment (DUET): The case of a CP child and his mother. Nordic Journal of Music Therapy, 19, 103-132, https://doi.org/10.1080/08098131.2010.500742. 
Gilliam, W., \& Mayes, L. (2005). Developmental assessment of infants and toddlers. In C. H. Zeanah (Ed.), Handbook of infant mental health (2nd ed., pp. 236-248). New York: The Guildford Press.

Gold, C., Wigram, T., \& Berger, E. (2001). The development of a research design to assess the effects of individual music therapy with mentally ill children and adolescents. Nordic Journal of Music Therapy, 10, 17-31, https://doi.org/10.1080/08098130109478014.

Golos, A., Sarid, M., Weill, M., \& Weintraub, N. (2011). Efficacy of and early intervention program for at-risk preschool boys: a two-group control study. The American Journal of Occupational Therapy, 65(4), 400-408.

Gross, W., Linden, U., \& Ostermann, T. (2010). Effects of music therapy in the treatment of children with dealyed speech development - results of a pilot study. BMC Complementary and Alternative Medicine, 10, 39, http://dx.doi.org/10.1186/1472-6882-10-39.

Guerrero, N., \& Turry, A. (2012). Nordoff-Robbins music therapy. An expressive and dynamic approach for young children on the autism spectrum. In P. Kern \& M. Humpal (Eds.), Early childhood music therapy and autism spectrum disorders. Developing potential in young children and their families (pp. 130-144). London, Philadelphia: Jessica Kingsley Publishers.

Hadley, S. J. (1996). A rationale for the use of songs with children undergoing bone marrow transplantation. Australian Journal of Music Therapy, 7, 16-27.

Hall, J. (2012). The school challenge. Combining the roles of music therapist and music teacher. In J. Tomlinson, P. Derrington, \& A. Oldfield (Eds.), Music therapy in schools. Working with children of all ages in mainstream and special education (pp. 75-87). London, Philadelphia: Jessica Kingsley Publishers.

Haslbeck, F. (2012). Music therapy for premature infants and their parents: an integrative review. Nordic Journal of Music Therapy, 21, 203-226, https://doi.org/10.1080/ 08098131.2011.648653.

Hayes, A., Lung, T., Wen, L., Baur, L., Rissel, C., \& Howard, K. (2014). Economic evaluation of "healthy beginnings" an early childhood intervention to prevent obesity. Obesity, 22(7), 1709-1715, https://doi.org/10.1002/oby.20747.

Hendon, C., \& Bohon, L. M. (2007). Hospitalized children's mood differences during play and music therapy. Child: Care, Health and Development, 34(2), 141-144, https://doi.org/ 10.1111/j.1365-2214.2007.00746.x.

Hibben, J. (1992). Music therapy in the treatment of families with young children. Music Therapy, 11(1), 28-44, https://doi.org/10.1093/mt/11.1.28.

Holck, U. (2004). Interaction themes in music therapy: definition and delimitation. Nordic Journal of Music Therapy, 13, 3-19, https://doi.org/10.1080/08098130409478094.

Hong, M., Hussey, D., \& Heng, M. (1998). Music therapy with children with severe emotional disturbances in a residential treatment setting. Music Therapy Perspectives, 16, 61-66, https://doi.org/10.1093/mtp/16.2.61.

Horvat, J., \& O’Neill, N. (2008). ‘Who is the therapy for?'Involving parent or carer in their child's music therapy. In A. Oldfield \& C. Flower (Eds.), Music therapy with children and their families (pp. 89-101). London, Philadelphia: Jessica Kingsley Publishers.

Humpal, M. (2012). Strategies and techniques. Making it happen for young children with autism spectrum disorders. In P. Kern \& M. Humpal (Eds.), Early childhood music therapy and autism spectrum disorders. Developing potential in young children and their families (pp. 162-180). London, Philadelphia: Jessica Kingsley Publishers.

Irgens-Moller, I. (1999). Music therapy in child psychiatry - a two-year project on a psychiatric unit for pre-school children. Nordic Journal of Music Therapy, 8, 73-76.

Jones, A., \& Oldfield, A. (1999). Sharing sessions with John. In J. Hibben (Ed.), Inside music therapy: Client experiences (pp. 165-171). Gilsum, NH: Barcelona Publishers.

Jonsdottir, V. (2002). Musicking in early intervention. Voices: A World Forum for Music Therapy, 2(2), https://doi.org/10.15845/voices.v2i2.86. 
Kelly, K. (2011). Supporting attachments in vulnerable families through an early intervention school-based group music therapy programme. In J. Edwards (Ed.), Music therapy and parent-infant bonding (pp. 22-41). Oxford, England: Oxford University press.

Kennely, J., Hamilton, L., \& Cross, J. (2001). The interface of music therapy and speech pathology in the rehabilitation of children with acquired brain injury. Australian Journal of Music Therapy, 12, 13-20.

Kern, P. (2006). Using embedded music therapy interventions to support outdoor play of young children with autism in an inclusive community-based child care program. Journal of Music Therapy, 43, 270-294, https://doi.org/10.1093/jmt/43.4.270.

Kern, P., \& Humpal, M. (Eds.). (2012). Early childhood music therapy and autism spectrum disorders. London, Philadelphia: Jessica Kingsley Publishers.

Kim, J., Wigram, T., \& Gold, C. (2008). The effects of improvisational music therapy on joint attention behaviors in autistic children: a randomized controlled study. Journal of Autism and Developmental Disorders, 38(9), 1758-1766, https://doi.org/10.1007/ s10803-008-0566-6.

Kim, J., Wigram, T., \& Gold, C. (2009). Emotional, motivational and interpersonal responsiveness of children with autism in improvisational music therapy. Autism, 13(4), 389-409, https://doi.org/10.1177/1362361309105660.

Layman, D. L., Hussey, D. L., \& Laing, S. J. (2002). Music therapy assessment for severely emotionally disturbed children: a pilot study. Journal of Music Therapy, 39, 164-187, https://doi.org/10.1093/jmt/39.3.164.

Lecourt, E. (1991). Off-beat music therapy: A psychoanalytic approach to autism. In K. Bruscia (Ed.), Case studies in music therapy (2nd ed., pp. 73-98). Gilsum, NH: Barcelona Publishers.

Ledger, A. (2011). Extending group music therapy to families in schools: a reflection on practical and professional aspects. In J. Edwards (Ed.), Music therapy and parent-infant bonding (pp. 127-140). Oxford, England: Oxford University press.

Leckman, J. F., \& March, J. S. (2011). Editorial: Developmental neuroscience comes of age. The Journal of Child Psychology and Psychiatry, 52(4), 333-338, https://doi.org/10.1111/ j.1469-7610.2011.02378.x.

Levinge, A. (1990). "The use of I and me": Music therapy with an autistic child. Journal of British Music Therapy, 4(2), 15-18, https://doi.org/10.1177/135945759000400204.

Levinge, A. (2011). "The first time ever I saw your face...": music therapy for depressed mothers and their infants. In J. Edwards (Ed.), Music therapy and parent-infant bonding (pp. 42-57). Oxford, England: Oxford University press.

Lim, H. (2012). Communication and language development. Implications for music therapy and autism spectrum disorders. In P. Kern \& M. Humpal (Eds.), Early childhood music therapy and autism spectrum disorders. Developing potential in young children and their families (pp. 199-213). London, Philadelphia: Jessica Kingsley Publishers.

Lim, H. (2010). Effect of "developmental speech and language training through music" on speech production in children with autism spectrum disorders. Journal of Music Therapy, 47, 2-26, https://doi.org/10.1093/jmt/47.1.2.

Lim, H. A., \& Draper, E. (2011). The effects of music therapy incorporated with applied behavior analysis verbal behavior approach for children with autism spectrum disorder. Journal of Music Therapy, 48, 532-550, https://doi.org/10.1093/jmt/48.4.532.

Lindenfelser, K. J., Grocke, D., \& McFerran, K. (2008). Bereaved parents' experiences of music therapy with their terminally ill child. Journal of Music Therapy, 45, 330-348, https://doi.org/10.1093/jmt/45.3.330.

Lindenfelser, K. J., Hense, C., \& McFerran, K. (2012). Music therapy in pediatric palliative care: family-centered care to enhance quality of life. American Journal of Hospice \& Palliative Medicine, 29(3), 219-226, https://doi.org/10.1177/1049909111429327.

Loewy, J. (2004). A clinical model of music therapy in the NICU. In M. Nöcker-Ribaupierre (Ed.), Music therapy for premature and newborn infants (pp. 159-175). Gilsum, NH: Barcelona Publishers. 
Loewy, J., Hallan, C., Friedman, E., \& Martinez, C. (2005). Sleep/sedation in children undergoing EEG testing: A comparison of chloral hydrate and music therapy. Journal of PeriAnesthesia Nursing, 20(5), 323-332, https://doi.org/10.1016/j.jopan.2005.08.001.

Lorenzato, K. I. (2005). Filling a need while making some noice. A music therapist's guide to pediatrics. London, Philadelphia: Jessica Kingsley Publishers.

Loth, H. (2008). Music therapy groups for families with a learning-disabled toddler. Bridging some gaps. In A. Oldfield \& C. Flower (Eds.), Music therapy with children and their families (pp. 53-69). London, Philadelphia: Jessica Kingsley Publishers.

MacKenzie, J., \& Hamlett, K. (2005). The music together program: addressing the needs of "well" families with young children. Australian Journal of Music Therapy, 16, 43-56.

MedicineNet.com. (n.d.). Retrieved from http://medicinenet.com

Muller, P., \& Warwick, A. (1997). Autistic children and music therapy. The effects of maternal involvement in therapy. In M. Heal \& T. Wigram (Eds.), Music Therapy in Health and Education (2nd ed., pp. 214-234). London, Philadelphia: Jessica Kingsley Publishers.

Nall, K., \& Everitt, E. (2005). From hospice to home: Music therapy outreach. In M. Pavlicevic (Ed.), Music therapy in children's hospices: Jessie's fund in action (pp. 147-158). London, Philadelphia: Jessica Kingsley Publishers.

Nicholson, J. M., Berthelsen, D., Abad, V., Williams, K., \& Bradley, J. (2008). Impact of music therapy to promote positive parenting and child development. Journal of Health and Psychology, 13(2), 226-238, https://doi.org/10.1177/1359105307086705.

Nicholson, J. M., Berthelsen, D., Williams, K. E., \& Abad, V. (2010). National study of early parenting intervention: Implementation differences on parenting and child outcomes. Prevention Science, 11(4), 360-370, https://doi.org/10.1007/s11121-010-0181-6.

O'Callaghan, C., Baron, A., Barry, P., \& Dun, B. (2011). Music's relevance for pediatric cancer patients: A constructivist and mosaic research approach. Support Care Cancer, 19(6), 779-788, https://doi.org/10.1007/s00520-010-0879-9.

O'Callaghan, C., \& Jordan, B. (2011). Music therapy supports parent-infant attachments: In families affected by life threatening cancer. In J. Edwards (Ed.), Music therapy and parentinfant bonding (pp. 191-207). Oxford, England: Oxford University press.

O'Neill, N. (2012). Open doors, open minds, open music! The development of music therapy provision in an assessment nursery. In J. Tomlinson, P. Derrington, \& A. Oldfield (Eds.), Music therapy in schools. Working with children of all ages in mainstream and special education (pp. 33-45). London, Philadelphia: Jessica Kingsley Publishers.

Oldfield, A. (2006). Interactive music therapy: A positive approach: music therapy at a child development centre. London, Philadelphia: Jessica Kingsley Publishers.

Oldfield, A. (1993). Music therapy with families. In M. Heal \& T. Wigram (Eds.), Music Therapy in Health and Education (pp. 46-54). London, Philadelphia: Jessica Kingsley Publishers.

Oldfield, A. (2011). Parents' perceptions of being in music therapy sessions with their children: What is our role as music therapists with parents? In J. Edwards (Ed.), Music therapy and parent-infant bonding (pp. 58-72). Oxford: Jessica Kingsley Publishers.

Oldfield, A. (2008). Working in partnership and supporting parents. Music therapy for preschool children and their parents at a child development centre. In A. Oldfield \& C. Flower (Eds.), Music therapy with children and their families (pp. 19-36). London, Philadelphia: Jessica Kingsley Publishers.

Oldfield, A., Adams, M., \& Bunce, L. (2003). An investigation into short-term music therapy with mothers and young children. British Journal of Music Therapy, 17(1), 26-45, https://doi.org/10.1177/135945750301700105.

Oldfield, A. (1991). Preverbal communication through music to overcome a child's language disorder. In K. Bruscia (Ed.), Case studies in music therapy (2nd ed., pp. 163-174). Gilsum, NH: Barcelona Publishers.

Oldfield, A. (2017). Music therapy with families in a psychiatric children's unit. In S. Lindahl Jacobsen \& G. Thompson (Eds.), Music therapy with families. Therapeutic approaches and theoretical perspectives (pp. 72-91). London, Philadelphia: Jessica Kingsley Publishers. 
Oldfield, A., \& Flower, C. (Eds.). (2008). Music therapy with children and their families. London, Philadelphia: Jessica Kingsley Publishers.

Pajulo, M., Pyykkönen, N., Kalland, M., Sinkkonen, J., Helenius, H., \& Punamäki, R-L. (2012). Substance-abusing mother-baby-pairs in residential treatment: Importance of pre-and postnatal reflective functioning. Infant Mental Health Journal, 33(1), 70-81, https://doi.org/ 10.1002/imhj.20342.

Papousek, M. (2011). Resilience, strengths, and regulatory capacities; Hidden resources in developmental disorders of infant mental health. Infant Mental Health Journal, 32(1), 29-46, https://doi.org/10.1002/imhj.20282.

Pasiali, V. (2012). Supporting parent-child interactions: Music therapy as an intervention for promoting mutually responsive orientation. Journal of Music Therapy, 49, 303-334, https://doi.org/10.1093/jmt/49.3.303.

Pasiali, V. (2012). Resilience, music therapy, and human adaption: Nurturing young children and families. Nordic Journal of Music Therapy, 21, 36-56, https://doi.org/10.1080/ 08098131.2011.571276.

Pavlicevic, M. (2005). Music therapy in children's hospices: Jessie's found in action. London, Philadelphia: Jessica Kingsley Publishers.

Pearce, C. (2011). A short introduction to promoting resilience in children. London, Philadelphia: Jessica Kingsley Publishers.

Perry, M. M. R. (2003). Relating improvisational music therapy with severely and multiply disabled children to communication development. Journal of Music Therapy, 40, 227-246, https://doi.org/10.1093/jmt/40.3.227.

Peters-Scheffera, N., Diddena, R., Korziliusd, H., \& Matsone, J. (2012). Cost comparison of early intensive behavioral intervention and treatment as usual for children with autism spectrum disorder in the Netherlands. Research in Developmental Disabilities, 33(6), 1763-1772, https://doi.org/10.1016/j.ridd.2012.04.006.

Philipp, D. A. (2012). Reflective family play: A model for whole family intervention in the infant and preschool clinical population. Infant Mental Health Journal, 33(6), 599-608, https://doi.org/10.1002/imhj.21342.

Randolph, J. J. (2009). A guide to writing the dissertation literature review. Practical Assessment, Research \& Evaluation, 14(13), 1-13.

Rees, C. (2005). Brief encounters. In M. Pavlicevic (Ed.), Music therapy in children's hospices: Jessie's fund in action (pp. 81-93). London, Philadelphia: Jessica Kingsley Publishers.

Register, D. (2001). The effects of an early intervention music curriculum on prereading/ writing. Journal of Music Therapy, 38, 239-248, https://doi.org/10.1093/jmt/38.3.239.

Register, D. (2004). The effects of live music groups versus an educational children's television program on the emergent literacy of young children. Journal of Music Therapy, 41, 2-27, https://doi.org/10.1093/jmt/41.1.2.

Register, D., \& Humpal, M. (2007). Using musical transitions in early childhood classrooms: Three case examples. Music Therapy Perspectives, 25, 25-31, https://doi.org/10.1093/mtp/ 25.1.25.

Reynolds, A., Temple, J., White, B., Ou, S-R., \& Robertson, D. (2011). Cost-benefit alysis of the child-parent center early education program. Child Development, 82(1), 379-404, https://doi.org/10.1111/j.1467-8624.2010.01563.x.

Robb, S. L. (2000). The effect of therapeutic music interventions on the behavior of hospitalized children in isolation: Developing a contextual support model of music therapy. Journal of Music Therapy, 37, 118-146, https://doi.org/10.1093/jmt/37.2.118.

Robb, S. L. (2003). Music interventions and group participation skills of preschoolers with visual impairments: raising questions about music, arousal, and attention. Journal of Music Therapy, 40(4), 266-282, https://doi.org/10.1093/jmt/40.4.266.

Roth, L., \& Sweatt, J. D. (2011). Epigenetic mechanisms and environmental shaping of the brain during sensitive periods of development. Journal of Child Psychology and Psychiatry, 54(4), 398-408, https://doi.org/https:/dx.doi.org/10.1111/j.1469-7610.2010.02282.x. 
Salas, J., \& Gonzales, D. (1991). Like singing with a bird: Improvisational music therapy with a blind four-year-old. In K. Bruscia (Ed.), Case studies in music therapy (2nd ed., pp. 17-27). Gilsum, NH: Barcelona Publishers.

Santelices, M., Guzmán, G., Aracena, M., Farkas, C., Armijo, I., Pérez-Salas, C., \& Borghini, A. (2011). Promoting secure attachment: evaluation of the effectiveness of an early intervention pilot programme with mother-infant dyads in Santiago, Chile. Child Care Health Development, 37(2), 203-210, https://doi.org/10.1111/j.1365-2214.2010.01161.x.

Sawyer, E., \& Burton, S. (2012). Building resilience in families under stress. Supporting families affected by parental substance misuse and/or mental health problems (2nd ed.). London, England: National Children's Bureau (NCB).

Sayre, J. M., Pianta, R. C., Marvin, R. S., \& Saft, E. W. (2001). Mothers'representations of relationships with their children: Relations with mother characteristics and feeding sensitivity. Journal of Pediatric Psychology, 26(6), 375-384, https://doi.org/10.1093/jpepsy/ 26.6.375.

Schwartz, E. (2008). Music therapy and early childhood: A developmental approach. Gilsum, NH: Barcelona Publishers.

Schwartz, E. K. (2011). Growing up in music: a journey through early childhood music develepment in music therapy. In A. Meadows (Ed.), Developments in music therapy practice: case study perspectives (pp. 70-85). Gilsum, NH: Barcelona Publishers.

Shoemark, H. (2006). Infant-directed singing as a vehicle for regulation rehearsal in the medically fragile full-term infant. Australian Journal of Music Therapy, 17, 54-63.

Shoemark, H. (2011). Translating "infant-directed singing" into a strategy for the hospitalized infant. In J. Edwards (Ed.), Music therapy and parent-infant bonding (pp. 161-178). Oxford, England: Oxford University press.

Shoemark, H. (2004). Family-centered music therapy for infants with complex medical and surgical needs. In M. Nöcker-Ribaupierre (Ed.), Music therapy for premature and newborn infants (pp. 141-157). Gilsum, NH: Barcelona Publishers.

Shoemark, H. (1996). Family-centred early intervention: Music therapy in the playground program. Australian Journal of Music Therapy, 7, 3-15.

Shoemark, H. (1999). Indications for the Inclusion of music therapy in the care of infants with bronchopulmonary dysplasia. In T. Wigram \& J. DeBacker (Eds.), Clinical applications of music therapy in cevelopmental disability, paediatrics and neurology (pp. 32-46). London, Philadelphia: Jessica Kingsley Publishers.

Shoemark, H., \& Dearn, T. (2008). Keeping parents at the centre of family centred music therapy with hospitalised infants. Australian Journal of Music Therapy, 19, 3-24.

Shoemark, H., \& Grocke, D. (2010). The markers of interplay between the music therapist and the high risk full term infant. Journal of Music Therapy, 47, 306-334, https://doi.org/ 10.1093/jmt/47.4.306.

Shonkoff, J. P., Lippitt, J. A., \& Cavanaugh, D. A. (2005). Early childhood policy: Implications for infant mental health. In C. H. Zeanah (Ed.), Handbook of infant mental health (2nd ed., pp. 503-518). New York: The Guildford Press.

Schore, A. (1994). Affect regulation and the origin of the self: the neurobiology of emotional development. New Jersey: Lawrence Erlbaum Associates, Inc., Publishers.

Schore, A. N. (2014). Early interpersonal neurobiological assessment of attachment and autistic spectrum disorders. Frontiers in Psychology, 5, 1049, https://doi.org/10.3389/ fpsyg.2014.01049.

Skewes, K., \& Thompson, G. (1998). The use of musical interactions to develop social skills in early intervention. Australian Journal of Music Therapy, 9(1), 35-44.

Solbakken, O. A., Sandvik, R., \& Monsen, J. T. (2011). Affect integration and reflective function: Clarification of central conceptual issues. Psychotherapy Research, 21(4), 482-496, https://doi.org/10.1080/10503307.2011.583696. 
Standley, J., Walworth, D., \& Nguyen, J. (2009). Effect of parent/child group music activities on toddler development: A pilot study. Music Therapy Perspectives, 27, 11-15,

https://doi.org/10.1093/mtp/27.1.11.

Standley, J. M., \& Hughes, J. E. (1996). Documenting developmentally appropriate objectives and benefits of a music therapy program for early intervention: A behavioral analysis. Music Therapy Pespectives, 14, 87-94, https://doi.org/10.1093/mtp/14.2.87.

Standley, J. M., \& Hughes, J. E. (1997). Evaluation of an early intervention music curriculum for enhancing prereading/writing Skills. Music Therapy Perspectives, 15, 79-5, https://doi.org/10.1093/mtp/15.2.79.

Standley, J. (2012). Music therapy research in the NICU: An updated meta-analysis. Neonatal Network, 31(5), 311-317, https://doi.org/10.1891/0730-0832.31.5.311.

Stern, D. (2010). Forms of vitality. Exploring dynamic experience in psychology, the arts, psychotherapy and development. Oxford, UK: Oxford University press.

Sussman, J. (2009). The effect of music on peer awareness in preschool age children with developmental disabilities. Journal of Music Therapy, 46, 53-68, https://doi.org/10.1093/ jmt/46.1.53.

Sweeney-Brown, C. (2005). Music and medicine: music therapy within a medical setting. In M. Pavlicevic (Ed.), Music therapy in children's hospices: Jessie's fund in action (pp. 48-61). London, Philadelphia: Jessica Kingsley Publishers.

Sweeney, C. (2003). "Couldn't put Humpty together again": Symbolic play with a terminally ill child. In S. J. Hadley (Ed.), Psychodynamic Music Therapy: Case Studies. Gilsum, NH: Barcelona Publishers.

Thompson, G. (2012). Family-centered music therapy in the home environment: Promoting interpersonal engagement between children with autism spectrum disorder and their parents. Music Therapy Perspectives, 30, 109-116, https://doi.org/10.1093/mtp/30.2.109.

Tomlinson, J., Derrington, P., \& Oldfield, A. (Eds.). (2012). Music therapy in schools. Working with children of all ages in mainstream and special education. London, Philadelphia: Jessica Kingsley Publishers.

Tomlinson, J. (2010). Music therapy for children with autism in an educational context. In V. Karkou (Ed.), Arts therapies in schools. Research and practice (pp. 231-242). London, Philadelphia: Jessica Kingsley Publishers.

Trevarthen, C., Aitken, K., Papoudi, D., \& Robarts, J. (1998). Children with autism. Diagnosis and interventions to meet their needs (2nd ed.). London, Philadelphia: Jessica Kingsley Publishers. pp. 172-202.

Trolldalen, G. (1997). Music therapy and Interplay. A music therapy project with mothers and children elucidated through the concept of "appreciative recognition.". Nordic Journal of Music Therapy, 6(1), 14-27.

Tuomi, K. (2017). Music therapy and theraplay. Creating, repairing and strengthening the attachment bond in foster and adoptive families. In S. Lindahl Jacobsen \& G. Thompson (Eds.), Music therapy with families. Therapeutic approaches and theoretical Perspectives (pp. 173-198). London, Philadelphia: Jessica Kingsley Publishers.

Voigt, M. (2003). Orff music therapy. Voices: A World Forum for Music Therapy, 3(3), https://doi.org/10.15845/voices.v3i3.134.

Walworth, D. (2005). Procedural-support music therapy in the healthcare setting: a costeffectiveness analysis. Journal of Pediatric Nursing, 20(4), 276-284, https://doi.org/ 10.1016/j.pedn.2005.02.016.

Walworth, D. (2009). Effects of developmental music groups for parents and premature or typical infants under two years on parental responsiveness and infant social development. Journal of Music Therapy, 46, 32-52, https://doi.org/10.1093/jmt/46.1.32.

Walworth, D., D, D., Register, D., \& Nguyen, J. (2009). Using the SCERTS model assessment tool to identify music therapy goals for clients with autism spectrum disorder. Journal of Music Therapy, 46, 204-216, https://doi.org/10.1093/jmt/46.3.204. 
Warnock, T. (2011). Voice and the self in improvised music therapy. British Journal of Music Therapy, 25(2), 32-47, https://doi.org/10.1177/135945751102500204.

Wigram, T. (1995). A model of assessment and differential diagnosis of handicap in children through the medium of music therapy. In T. Wigram, B. Saperston, \& R. West (Eds.), The art and science of music therapy: A handbook (pp. 181-195). Chur: Harwood Academic Publishers.

Wildman, C. (1995). Music Therapist as case study: Examining counter-transference with a young child. Nordic Journal of Music Therapy, 4, 3-10.

Williams, K., \& Abad, V. (2005). Reflections on music therapy with indigenous families: Cultural learning put into practice. Australian Journal of Music Therapy, 16(1), 60-69.

Williams, K. E., Berthelsen, D., Nicholson, J. M., Walker, S., \& Abad, V. (2012). The effectiveness of a short-term group music therapy intervention for parents who have a child with a disability. Journal of Music Therapy, 49, 23-44, https://doi.org/10.1093/jmt/49.1.23.

Woodward, A. (2004). Music therapy for autistic children and their families: a creative spectrum. British Journal of Music Therapy, 18(1), 8-14, https://doi.org/10.1177/ 135945750401800103.

Wylie, M. E. (1996). A case study to promote hand use in children with rett syndrome. Music Therapy Perspectives, 14, 83-86, https://doi.org/10.1093/mtp/14.2.83. 